\title{
Do all BPS black hole microstates carry zero angular momentum?
}

\author{
Abhishek Chowdhury, ${ }^{a}$ Richard S. Garavuso, ${ }^{b}$ Swapnamay Mondal ${ }^{a}$ and Ashoke Sen ${ }^{a}$ \\ ${ }^{a}$ Harish-Chandra Research Institute, \\ Chhatnag Road, Jhusi, Allahabad 211019, India \\ ${ }^{b}$ Physical Sciences, Kingsborough Community College, The City University of New York, \\ 2001 Oriental Boulevard, Brooklyn, NY 11235-2398, U.S.A. \\ E-mail: abhishek@hri.res.in, richard.garavuso@kbcc.cuny .edu, \\ swapno@hri.res.in, sen@hri.res.in
}

ABSTRACT: From the analysis of the near horizon geometry and supersymmetry algebra it has been argued that all the microstates of single centered BPS black holes with four unbroken supersymmetries carry zero angular momentum in the region of the moduli space where the black hole description is valid. A stronger form of the conjecture would be that the result holds for any sufficiently generic point in the moduli space. In this paper we set out to test this conjecture for a class of black hole microstates in type II string theory on $T^{6}$, represented by four stacks of D-branes wrapped on various cycles of $T^{6}$. For this system the above conjecture translates to the statement that the moduli space of classical vacua must be a collection of points. Explicit analysis of systems carrying a low number of D-branes supports this conjecture.

KeYwords: Black Holes in String Theory, D-branes

ArXiv EPRINT: 1511.06978 


\section{Contents}

1 Introduction 1

2 The system 4

3 Supersymmetric ground states for $N=1 \quad 9$

3.1 F-term equations 9

$\begin{array}{lll}3.2 & \text { D-term equations } & 10\end{array}$

$3.3 X_{i}^{(k)}$ dependent terms $\quad 11$

3.4 Gauss' law constraint 11

$4 \quad$ Supersymmetric ground states for $N=2 \quad 12$

$\begin{array}{lll}4.1 & \text { F-term equations } & 12\end{array}$

$\begin{array}{lll}4.2 & \text { D-term equations } & 13\end{array}$

$4.3 X_{i}^{(k)}$ dependent terms 14

$\begin{array}{lll}4.4 & \text { Gauss' law constraint } & 15\end{array}$

$5 \quad$ Supersymmetric ground states for $N=3 \quad 15$

6 Conclusion 16

$\begin{array}{ll}\text { A Normalization of } Z-Z-Z \text { coupling } & 17\end{array}$

B Explicit solutions for $N=2 \quad 25$

\section{Introduction}

Based on the analysis of the near horizon geometry of a BPS black hole it has been argued that as long as the black hole carries four unbroken supersymmetries, the microstates of single centered black holes will all carry zero angular momentum $[1,2] .{ }^{1}$ Since the

\footnotetext{
${ }^{1}$ This statement requires some qualification since all BPS black holes form a representation of the supersymmetry algebra carrying many different spins. This arises from the quantization of the fermion zero modes associated with broken supersymmetry. In the black hole description these modes live outside the horizon. On the other hand, the property of zero angular momentum refers to the microstates associated with the black hole horizon. Therefore the correct statement is that the microstates of single centered BPS black holes are given by the tensor product of the elementary BPS supermultiplet, obtained by quantization of the goldstino fermion zero modes, and singlet representation of the $\mathrm{SU}(2)$ rotation group. For black holes in five dimensions a further qualification is needed - the black hole microstates are singlets under one of the two $\mathrm{SU}(2)$ 's - the one that forms a subgroup of the $\mathrm{SU}(1,1 \mid 2)$ supergroup describing symmetries of the near horizon geometry. This complication will not affect our analysis since we shall focus on black holes in $3+1$ dimensions.
} 
counting of black hole microstates is normally done in a regime that is different from the regime in which the black hole description is valid, it is difficult to test this conjecture directly. Indirect tests of this have been performed by examining the BPS index - given by number of states weighted by $(-1)^{2 J_{3}}$ after factoring out the goldstino fermion zero mode contributions - which is expected to be independent of the moduli. On the black hole side if all the microstates carry zero angular momentum, then the BPS index will be positive. This implies that on the microscopic side also we must always have positive index. This has been tested in many examples [3, 4], and so far no counterexample has been found, even though the microscopic counting typically gives states carrying different angular momenta (even after factoring out the the goldstino zero mode contribution), some of which give positive contribution to the index and others give negative contribution.

If this conjecture is correct, then one might wonder at what point in the moduli space does the spectrum of BPS states change from having various different angular momenta to only zero angular momentum? A natural conjecture - which we shall call the strong form of the zero angular momentum conjecture - will be that this happens at any sufficiently generic point in the moduli space, even in regions where the system is weakly coupled and the microscopic description is valid. This is not in direct conflict with the results for the microscopic spectrum described at the end of the previous paragraph, since typically the microscopic spectrum is computed not only by setting the string coupling to be weak, but also by setting several other moduli to special values where the quantum mechanics describing the microstates simplifies.

A possible complication in testing this conjecture is that it refers to the microstates of single centered black holes. Even in regions where the black hole description is valid, typically there are multi-centered BPS black hole configurations contributing to this index, and these typically carry non-zero angular momentum. In $\mathcal{N}=2$ supersymmetric theories in $3+1$ dimensions the contribution from the multi-centered black holes is very complicated [5] and it is not easy to disentangle it from the contribution from single centered black holes, although some progress has been made on this front [6]. In this context the results of [7-11] can be taken as providing partial support to this conjecture. The situation is somewhat better in $\mathcal{N}=4$ supersymmetric theories where the contribution from multicentered black holes are better understood [12-15]. But the situation is best for $\mathcal{N}=8$ supersymmetric theories, where for the charge configuration of the kind for which we have black holes, there is no contribution to the index from multi-centered black holes [16] and in fact all multi-centered BPS states are expected to disappear at a generic point in the moduli space. Therefore in this case the strong form of the conjecture will imply that at a generic point in the moduli space, all the microstates must carry zero angular momentum.

In a previous paper [17] we began to test this conjecture by considering a system of four stacks of D-branes in type II string theory of $T^{6}$. If we denote by $x^{4}, \ldots, x^{9}$ the coordinates along $T^{6}$, then in an appropriate duality frame the general system considered in [17] can be represented as $N_{1}$ D2-branes wrapped along the 4-5 directions, $N_{2}$ D2-branes wrapped along the 6-7 directions, $N_{3}$ D2-branes wrapped along the 8-9 directions and $N_{4}$ D6-branes wrapped along the 4-5-6-7-8-9 directions. We keep the moduli sufficiently generic by switching on generic values of the constant metric and NS-NS 2-form background along $T^{6}$. The 
low energy dynamics of the system was shown to describe a specific quantum mechanical system, obtained from the dimensional reduction of an $\mathcal{N}=1$ supersymmetric field theory in $3+1$ dimensions to $0+1$ dimensions. The moduli space describing the classical supersymmetric solutions ${ }^{2}$ corresponds to gauge inequivalent solutions to the F- and D-term constraints of the theory, and the quantum BPS states are given by harmonic forms on this moduli space [18]. By considering the simplest system in which we have one D-brane in each of the four stacks, it was found that except for trivial flat directions associated with translation along the non-compact directions as well as along $T^{6}$ and dual $T^{6}$, the moduli space is a collection of 12 points. The quantization of the translational zero modes produces states carrying different momenta along the non-compact directions and along $T^{6}$ and also different fundamental string winding charges along $T^{6}$. Once these quantum numbers are fixed we get a unique state from quantization of the bosonic zero modes. The fermionic partners of these bosonic zero modes are the 28 goldstino fermion zero modes, whose quantization produces the basic BPS supermultiplet. The fact that after factoring out the zero mode contributions the moduli space becomes a collection of isolated points shows that the BPS ground state associated with each solution is unique, and hence must be singlets of the $\mathrm{SU}(2)$ rotation group. ${ }^{3}$ The 12 isolated configurations then lead to 12 BPS states, each carrying zero angular momentum. This is consistent with the strong conjecture stated above. This also shows that the BPS index of this system is 12. This is in perfect agreement with the result of the computation of the index for the same system in a dual description [20,21].

In this paper we extend the analysis of [17] to the cases where the number of D6branes in the stack is 2 or 3 . Again we find that for generic constant background values of metric and NS-NS 2-form fields, the moduli space of classical supersymmetric configurations consists of a discrete set of points after factoring out the zero mode contribution. This in turn shows that after factoring out the basic BPS supermultiplet we are left with a set of singlet representations of the $\mathrm{SU}(2)$ rotation group: 56 for the case of 2 D6-branes and 208 for the case of 3 D6-branes. This is again consistent with the strong form of the conjecture stated above. Furthermore since all states are SU(2) singlets, the BPS indices associated with these states are also given by 56 and 208, respectively. This agrees precisely with the index computed in a dual description [21]. However in [21] the counting was carried out at a non-generic point in the moduli space, and the spectrum contained BPS states carrying different angular momenta. The index was the final result after imperfect cancellation between contributions from bosonic and fermionic states. In contrast here all states carry zero angular momentum and give positive contribution to the index.

The rest of the paper is organized as follows. In section 2 we describe the system under study, and review the results of [17] for the supersymmetric quantum mechanics describing the low energy dynamics of this system. In section 3 we describe in detail the steps we follow for counting the number of BPS states consisting of a system of one D2-brane along 4-5 directions, one D2-brane along 6-7 directions, one D2-brane along 8-9 directions and one D6-brane along 4-5-6-7-8-9 directions. In section 4 we describe the analysis for a

\footnotetext{
${ }^{2}$ This should not be confused with the moduli space describing background closed string fields which are kept at generic values.

${ }^{3}$ A similar result for the D0-D4 system was found in [19].
} 
system consisting of one D2-brane along 4-5 directions, one D2-brane along 6-7 directions, one D2-brane along 8-9 directions and two D6-branes along 4-5-6-7-8-9 directions. In section 5 we briefly describe the analysis for three D6-branes along 4-5-6-7-8-9 directions, leaving the number of D2-branes unchanged. We conclude in section 6 with a discussion on the possible implications of our results in general, and in particular for the fuzzball program. In appendix A we carefully fix the signs and normalizations of various terms in the superpotential - the analysis of [17] was insensitive to these choices but they seem to be relevant for analyzing the general case with multiple D-branes in one or more stacks. In this we fix the normalization by explicit string theory computation and the signs by various symmetry requirements. We are however left with a twofold ambiguity in the choice of sign, only one of which yields the correct answer in different cases we have studied. While a careful string theory computation should be able to resolve this ambiguity, we have not done this. In appendix B we give explicit results for the 56 solutions in the special case when one of the stacks contains two D-branes with the other stacks containing one D-brane each.

\section{The system}

We consider type IIA string theory on $T^{6}$ labelled by the coordinates $x^{4}, \ldots, x^{9}$ and in this theory we take a system containing one D2-brane wrapped along 4-5 directions, one D2-brane wrapped along 6-7 directions, one D2-brane wrapped along 8-9 directions and $N$ D6-branes wrapped along 4-5-6-7-8-9 directions, where $N$ takes values 1,2 and $3 .{ }^{4}$ As in [17] we shall work at a generic point in the moduli space at which constant metric and 2 -form backgrounds are turned on along $T^{6}$. The supersymmetric quantum mechanics describing this system was constructed in [17]. Since this system has four unbroken supercharges the same as for $\mathcal{N}=1$ supersymmetric theories in four dimensions - we shall use the language of four dimensional theories to organize the fields and terms in the Lagrangian. However since we are really considering a quantum mechanical system, we ignore all spatial derivative terms in the action.

We begin by listing the field content of this $0+1$ dimensional field theory. We shall focus only on the bosons since the fermionic field content is determined by supersymmetry. The four dimensional theory can be regarded as an $\mathcal{N}=1$ supersymmetric theory with gauge group $\mathrm{U}(1) \times \mathrm{U}(1) \times \mathrm{U}(1) \times \mathrm{U}(N)$ with additional chiral multiplets. We denote by $X_{i}^{(k)}$ for $1 \leq k \leq 4,1 \leq i \leq 3$ the $i$-th spatial component of these four sets of gauge fields. $k=1,2,3$ will stand for the $\mathrm{U}(1)$ factors and $k=4$ will stand for the $\mathrm{U}(N)$ factor. ${ }^{5}$ Therefore $X_{i}^{(4)}$ denotes an $N \times N$ Hermitian matrix for each $i$ whereas $X_{i}^{(k)}$ for $1 \leq k \leq 3$, $1 \leq i \leq 3$ are real numbers. Physically the $X_{i}^{(k)}$ for $1 \leq k \leq 3$ describe the position of the $k$-th D2-brane along the three non-compact directions and the diagonal elements of $X_{i}^{(4)}$

\footnotetext{
${ }^{4}$ Alternatively, by making T-duality transformations along each of the six circles we can regard this as a system of one D4-brane along each of the three 4-cycles 4567, 6789 and 4589, and $N$ D0-branes. Under this transformation the quantum theory will remain the same, but the physical interpretation of the different variables given below will differ, with positions and Wilson lines getting exchanged.

${ }^{5}$ There are also non-dynamical fields $X_{0}^{(k)}$ which implement the gauge invariance constraints. Their effect will be discussed later.
} 
label the positions of the $N$ D6-branes along the non-compact directions. The off-diagonal components of $X_{i}^{(4)}$ arise from open strings stretched between the D6-branes. The rest of the bosonic fields can be organized into chiral multiplet scalars. First of all, for each of the four stacks of D-branes we have three chiral multiplets in the adjoint representation. We shall denote them by $\Phi_{i}^{(k)}$ for $1 \leq k \leq 4,1 \leq i \leq 3$. $\Phi_{i}^{(k)}$ for $1 \leq k \leq 3$ describe $1 \times 1$ complex matrices whereas $\Phi_{i}^{(4)}$ will describe an $N \times N$ complex matrix, transforming in the adjoint representation of the respective gauge groups. Physically the $\Phi_{i}^{(k)}$ 's for $1 \leq k \leq 3$ label the position of the $k$-th D2-brane along the directions of $T^{6}$ transverse to the D2-brane, and also the Wilson lines on the $k$-th D2-brane along directions tangential to the D2-brane. On the other hand the diagonal elements of $\Phi_{i}^{(4)}$ label the Wilson lines along the D6-branes. The off-diagonal components of $\Phi_{i}^{(4)}$ arise from open strings stretched between the D6branes. Besides these fields, for every pair of D-brane stacks labelled by $(k, \ell)$ we have two chiral superfields $Z^{(k \ell)}$ and $Z^{(\ell k)}$ arising from open strings stretched between the two D-brane stacks, transforming respectively in the bi-fundamental and anti-bi-fundamental representation of the corresponding gauge groups. Therefore $Z^{(k \ell)}$ for $1 \leq k, \ell \leq 3$ are $1 \times 1$ matrices, $Z^{(k 4)}$ for $1 \leq k \leq 3$ are $1 \times N$ matrices and $Z^{(4 k)}$ for $1 \leq k \leq 3$ are $N \times 1$ matrices. Under the rotation group $\mathrm{SU}(2)$ acting on the non-compact directions, $X_{i}^{(k)}$ 's transform as vectors whereas $\Phi_{i}^{(k)}$ 's and $Z^{(k \ell)}$ 's transform as scalars.

The potential involving these fields receives contributions from several sources. First of all we have several terms in the superpotential. ${ }^{6}$ They are given by [17]

$$
\begin{aligned}
\mathcal{W}_{1}=\sqrt{2}[ & \sum_{k, \ell, m=1}^{3} \varepsilon^{k \ell m} \operatorname{Tr}\left(\Phi_{m}^{(k)} Z^{(k \ell)} Z^{(\ell k)}\right)+\sum_{k=1}^{3} \operatorname{Tr}\left(Z^{(4 k)} \Phi_{k}^{(k)} Z^{(k 4)}\right) \\
& \left.-\sum_{k=1}^{3} \operatorname{Tr}\left(\Phi_{k}^{(4)} Z^{(4 k)} Z^{(k 4)}\right)\right] \\
=\sqrt{2}\left[\left(\Phi_{3}^{(1)}-\Phi_{3}^{(2)}\right) Z^{(21)} Z^{(12)}+\left(\Phi_{1}^{(2)}-\Phi_{1}^{(3)}\right) Z^{(32)} Z^{(23)}+\left(\Phi_{2}^{(3)}-\Phi_{2}^{(1)}\right) Z^{(31)} Z^{(13)}\right. & \\
& +\operatorname{Tr}\left(\left(\Phi_{1}^{(1)} I_{N}-\Phi_{1}^{(4)}\right) Z^{(41)} Z^{(14)}\right)+\operatorname{Tr}\left(\left(\Phi_{2}^{(2)} I_{N}-\Phi_{2}^{(4)}\right) Z^{(42)} Z^{(24)}\right) \\
& \left.+\operatorname{Tr}\left(\left(\Phi_{3}^{(3)} I_{N}-\Phi_{3}^{(4)}\right) Z^{(43)} Z^{(34)}\right)\right], \\
\mathcal{W}_{2}=\sqrt{2} & {\left[\sum_{\substack{k, \ell, m=1 \\
k<\ell, m ; \ell \neq m}}(-1)^{\delta_{k 1} \delta_{\ell 3} \delta_{m 4}} \operatorname{Tr}\left(Z^{(k \ell)} Z^{(\ell m)} Z^{(m k)}\right)\right] } \\
=\sqrt{2}[ & Z^{(31)} Z^{(12)} Z^{(23)}+Z^{(13)} Z^{(32)} Z^{(21)}+\operatorname{Tr}\left(Z^{(12)} Z^{(24)} Z^{(41)}\right)+\operatorname{Tr}\left(Z^{(42)} Z^{(21)} Z^{(14)}\right) \\
& -\operatorname{Tr}\left(Z^{(13)} Z^{(34)} Z^{(41)}\right)+\operatorname{Tr}\left(Z^{(31)} Z^{(14)} Z^{(43)}\right)+\operatorname{Tr}\left(Z^{(34)} Z^{(42)} Z^{(23)}\right)
\end{aligned}
$$

\footnotetext{
${ }^{6}$ The superpotential may contain other terms, e.g. terms quartic in $Z^{(k \ell)}$ 's. However if we consider the limit when all the $c^{(k \ell)}$ 's and $c^{(k)}$ 's are small, say of order $\lambda$ for some small parameter $\lambda$, then the solutions to the F- and D-term equations occur at $Z^{(k \ell)}, \Phi_{i}^{(k)} \sim \sqrt{\lambda}$. In this limit the effect of the other terms in the superpotential can be ignored. This limit also allows us to ignore the fact that the $\Phi_{i}^{(k)}$ fields have periodic identification.
} 


$$
\begin{gathered}
\left.+\operatorname{Tr}\left(Z^{(43)} Z^{(32)} Z^{(24)}\right)\right] \\
\mathcal{W}_{3}=\sqrt{2}\left[\sum_{k, \ell, m=1}^{3} c^{(k \ell)} \varepsilon^{k \ell m} \operatorname{Tr}\left(\Phi_{m}^{(k)}\right)+\sum_{k=1}^{3} c^{(k 4)}\left[N_{4} \operatorname{Tr}\left(\Phi_{k}^{(k)}\right)-\operatorname{Tr}\left(\Phi_{k}^{(4)}\right)\right]\right] \\
=\sqrt{2}\left[c^{(12)}\left(\Phi_{3}^{(1)}-\Phi_{3}^{(2)}\right)+c^{(23)}\left(\Phi_{1}^{(2)}-\Phi_{1}^{(3)}\right)+c^{(13)}\left(\Phi_{2}^{(3)}-\Phi_{2}^{(1)}\right)+c^{(14)} \operatorname{Tr}\left(\Phi_{1}^{(1)} I_{N}-\Phi_{1}^{(4)}\right)\right. \\
\left.+c^{(24)} \operatorname{Tr}\left(\Phi_{2}^{(2)} I_{N}-\Phi_{2}^{(4)}\right)+c^{(34)} \operatorname{Tr}\left(\Phi_{3}^{(3)} I_{N}-\Phi_{3}^{(4)}\right)\right]
\end{gathered}
$$

and

$$
\mathcal{W}_{4}=-\sqrt{2} \operatorname{Tr}\left(\Phi_{1}^{(4)}\left[\Phi_{2}^{(4)}, \Phi_{3}^{(4)}\right]\right)=-\sqrt{2} \operatorname{Tr}\left(\Phi_{1}^{(4)} \Phi_{2}^{(4)} \Phi_{3}^{(4)}-\Phi_{1}^{(4)} \Phi_{3}^{(4)} \Phi_{2}^{(4)}\right),
$$

where $c^{(k \ell)}=c^{(\ell k)}$ for $1 \leq k<\ell \leq 4$ are parameters whose values are determined in terms of the background metric and 2-form fields [17], and $I_{p}$ denotes the $p \times p$ identity matrix. There are some differences from the form of the superpotential given in [17]. First of all in [17] the overall coefficient of $\mathcal{W}_{2}$ was undetermined. For $N=1$ this constant can be scaled away by appropriate scaling of the fields $Z^{(k \ell)}, \Phi_{i}^{(k)}$ and the parameters $c^{(k \ell)}$, and so its value was not needed. However for $N \geq 2$ this can no longer be done without changing the coefficient of the cubic $\Phi-\Phi-\Phi$ coupling. Since the result depends on the choice of this coefficient, we have fixed it from explicit string theory computation described in appendix A. Second, we have a strange sign $(-1)^{\delta_{k 1} \delta_{\ell 3} \delta_{m 4}}$ in the expression for $\mathcal{W}_{2}$ which is responsible for the minus sign in front of the $\operatorname{Tr}\left(Z^{(13)} Z^{(34)} Z^{(41)}\right)$ term. This sign was missed in [17] but must be there for symmetry reasons. This has also been explained in appendix A. ${ }^{7}$ In appendix A we also discuss generalization of (2.1)-(2.4) to the case where we have an arbitrary number of D-branes in each of the four stacks.

The F-term potential is given by

$$
V_{F}=\sum_{\alpha}\left|\frac{\partial \mathcal{W}}{\partial \varphi_{\alpha}}\right|^{2}
$$

where

$$
\mathcal{W}=\mathcal{W}_{1}+\mathcal{W}_{2}+\mathcal{W}_{3}+\mathcal{W}_{4}
$$

and $\left\{\varphi_{\alpha}\right\}$ denotes the set of all the chiral superfields.

Besides the $F$-term potential, there is a D-term potential given by

$$
V_{D}=\frac{1}{2} \sum_{k=1}^{4} \operatorname{Tr}\left[\left(\sum_{\substack{\ell=1 \\ \ell \neq k}}^{4} Z^{(k \ell)} Z^{(k \ell) \dagger}-\sum_{\substack{\ell=1 \\ \ell \neq k}}^{4} Z^{(\ell k) \dagger} Z^{(\ell k)}+\sum_{i=1}^{3}\left[\Phi_{i}^{(k)}, \Phi_{i}^{(k) \dagger}\right]-c^{(k)} I_{N_{k}}\right)^{2}\right],
$$

where $N_{1}=N_{2}=N_{3}=1$ and $N_{4}=N$. The FI parameters $c^{(k)}$ are also determined from the background values of the 2-form field and satisfy

$$
\sum_{k=1}^{4} c^{(k)} N_{k}=0
$$

\footnotetext{
${ }^{7}$ As discussed in section 3, the result for the number of states for $N=1$, obtained in [17], is not affected by this change of sign.
} 
Finally the dimensional reduction of the coupling of the gauge fields (whose spatial components are denoted by $X_{i}^{(k)}$ ) to chiral multiplets leads to the potential

$$
\begin{aligned}
V_{\text {gauge }}= & \sum_{k=1}^{4} \sum_{\substack{\ell=1 \\
\ell \neq k}}^{4} \sum_{i=1}^{3} \operatorname{Tr}\left[\left(X_{i}^{(k)} Z^{(k \ell)}-Z^{(k \ell)} X_{i}^{(\ell)}\right)^{\dagger}\left(X_{i}^{(k)} Z^{(k \ell)}-Z^{(k \ell)} X_{i}^{(\ell)}\right)\right] \\
& +\sum_{k=1}^{4} \sum_{i, j=1}^{3} \operatorname{Tr}\left(\left[X_{i}^{(k)}, \Phi_{j}^{(k)}\right]^{\dagger}\left[X_{i}^{(k)}, \Phi_{j}^{(k)}\right]\right)+\frac{1}{4} \sum_{k=1}^{4} \sum_{i, j=1}^{3} \operatorname{Tr}\left(\left[X_{i}^{(k)}, X_{j}^{(k)}\right]^{\dagger}\left[X_{i}^{(k)}, X_{j}^{(k)}\right]\right) .
\end{aligned}
$$

Therefore the total potential is given by

$$
V=V_{F}+V_{D}+V_{\text {gauge }}
$$

The potential given above has a shift symmetry

$$
\begin{aligned}
& \Phi_{m}^{(k)} \rightarrow \Phi_{m}^{(k)}+\xi_{m}, \quad \text { for } \quad 1 \leq k \leq 3, \quad k \neq m, \quad 1 \leq m \leq 3, \\
& \Phi_{k}^{(k)} \rightarrow \Phi_{k}^{(k)}+\zeta_{k}, \quad \Phi_{k}^{(4)} \rightarrow \Phi_{k}^{(4)}+\zeta_{k} I_{N}, \quad \text { for } \quad 1 \leq k \leq 3, \\
& X_{i}^{(k)} \rightarrow X_{i}^{(k)}+a_{i}, \quad X_{i}^{(4)} \rightarrow X_{i}^{(4)}+a_{i} I_{N}, \quad \text { for } \quad 1 \leq i \leq 3, \quad 1 \leq k \leq 3,
\end{aligned}
$$

where $\left\{\xi_{m}\right\}$ and $\left\{\zeta_{k}\right\}$ are arbitrary complex parameters and $\left\{a_{i}\right\}$ are arbitrary real parameters. These shift symmetries generate six complex translations along the compact directions and their duals and three real translations along the non-compact directions.

Our strategy for determining the spectrum of BPS states will be to regard the low energy dynamics of the system as the motion of a superparticle moving on the classical vacuum manifold defined by the space of $V=0$ configurations, and then quantize the system and find its supersymmetric ground states. Now the shift symmetries (2.11) generate flat directions of the potential $V$. Quantization of the bosonic zero modes associated with these flat directions leads to a unique ground state if we restrict to the sector carrying zero momentum and winding along the internal directions and zero momentum along the non-compact directions. There are also associated fermionic zero modes describing the goldstino modes corresponding to $32-4=28$ broken supersymmetries. Quantization of these fermion zero modes produces the supermultiplet describing $1 / 8$ BPS states of $\mathcal{N}=8$ supersymmetric string theory but has no other effect on the rest of the system. The BPS spectrum is given by a tensor product of this basic supermultiplet with some (possibly reducible) representation of the rotation group $\mathrm{SU}(2)$. Our goal will be to determine which representation of $\mathrm{SU}(2)$ is tensored with the basic supermultiplet.

The information about the $\mathrm{SU}(2)$ representation with which the supermultiplet is tensored is contained in the character $P(y) \equiv \operatorname{Tr}\left(y^{2 J_{3}}\right)$ of the representation. $P(y)$ is computed as follows. We shall show at the end of appendix A that the vanishing of $V_{\text {gauge }}$ given in (2.9) requires all the $X_{i}^{(k)}$ 's to vanish (up to the shift symmetry described in the last line of (2.11)). If the gauge inequivalent solutions to the $V_{F}=V_{D}=0$ condition generate a manifold $\mathcal{M}$ of complex dimension $d$ after factoring out the flat directions of the potential associated with the symmetries given in (2.11), and setting the $X_{i}^{(k)}$ 's to zero, then the BPS states of the system are in one to one correspondence with the harmonic forms on $\mathcal{M}$, and the rotational $\mathrm{SU}(2)$ is identified with the Lefschetz $\mathrm{SU}(2)$ acting on these forms 
(see e.g. [7, 22]). Therefore if $b_{p}$ denotes the $p$-th betti number of $\mathcal{M}$ and $d$ denotes the complex dimension of $\mathcal{M}$, then we have ${ }^{8}$

$$
P(y)=\sum_{p} b_{p} y^{p-d} .
$$

If $\mathcal{M}$ contains several components then we have to add up the contribution from various components to get the total $P(y)$. The BPS index (which is the 14-th helicity trace $-B_{14}[23,24]$ from the space-time viewpoint) is given by $\operatorname{Tr}(-1)^{2 J_{3}}$, with the trace running over the states with which the basic BPS supermultiplet is tensored to get the full spectrum of BPS states. Therefore it is given by $P(-1)$, which, according to $(2.12)$, is $(-1)^{d}$ times the Euler character of $\mathcal{M}$.

The conjecture that all BPS states carry zero angular momentum now translates to the requirement that $P(y)$ is $y$-independent, i.e. the subspace $\mathcal{M}$ consists of isolated points. In this case the BPS index $P(-1)$ is equal to the degeneracy $P(1)$ and just counts the number of gauge inequivalent solutions to the $V=0$ condition.

We conclude this section by giving the expected result for the index from analysis of the spectrum in a dual description containing a D1-D5-KK monopole state carrying momentum along the common circle shared by the world-volume of the D1-brane, D5-brane and the KK monopole. Let us denote by $\hat{c}(u)$ the numbers appearing in the expansion

$$
-\vartheta_{1}(z \mid \tau)^{2} \eta(\tau)^{-6} \equiv \sum_{k, l} \hat{c}\left(4 k-l^{2}\right) e^{2 \pi i(k \tau+l z)},
$$

where $\vartheta_{1}(z \mid \tau)$ and $\eta(\tau)$ are respectively the odd Jacobi theta function and the Dedekind eta function. Then the expected result for the index $P(-1)$ is $[21]^{9}$

$$
-\hat{c}(4 N) \text {. }
$$

Explicit computation gives

$$
-\hat{c}(4)=12, \quad-\hat{c}(8)=56, \quad-\hat{c}(12)=208, \quad-\hat{c}(16)=684, \quad \cdots
$$

In the previous paper [17] we carried out the analysis for $N=1$ and found 12 isolated solutions to the $V=0$ equation. Using (2.14) and (2.15) we see that this is in perfect agreement with the results from the dual description. In this paper we shall carry out the computation for $N=1,2$ and 3 using a slightly different method. The reanalysis of the $N=1$ case is important since the sign of the $\operatorname{Tr}\left(Z^{(13)} Z^{(34)} Z^{(41)}\right)$ term in $\mathcal{W}_{2}$ given in $(2.2)$ differs from that used in [17]. It turns out however that the result remains unchanged.

\footnotetext{
${ }^{8}$ Intuitively this identification can be understood as follows. Since the vacuum manifold has $X_{i}^{(k)}=0$ for all $k, i$, the moduli space is spanned by the scalars. The fermionic partners of the scalars take values in the tangent space of $\mathcal{M}$ - in fact for each tangent vector there are two massless fermions which we can denote by $\psi^{a}$ and $\psi^{a \dagger}$ where $a$ labels independent tangent vectors. We can choose $\psi^{a}$ and $\psi^{a \dagger}$ such that $\psi^{a \dagger}$ has $J_{3}=$ $1 / 2$ and $\psi^{a}$ has $J_{3}=-1 / 2$. Now we can begin with the states annihilated by all the $\psi^{a}$ 's, identify them as the zero forms on $\mathcal{M}$, and build the total space of states by applying $\psi^{a \dagger}$ 's on this state. This space is isomorphic to the space of forms on $\mathcal{M}$, and the BPS condition translates to these forms being harmonic. In this notation we see that the $p$-forms carry $J_{3}$ eigenvalue $(p-d) / 2$, where the shift $-d / 2$ is the $J_{3}$ eigenvalue of the zero forms, and is necessary to ensure that the states form a representation of SU(2). This leads to (2.12).

${ }^{9}$ Various macroscopic tests of this formula beyond the one provided by the Bekenstein-Hawking formula have been carried out in [25-29].
} 


\section{Supersymmetric ground states for $N=1$}

Our goal is to determine the manifold $\mathcal{M}$ parametrizing the gauge inequivalent solutions to the equation $V=0$. Since the potential is a sum of positive semi-definite terms, this in turn requires that each term in the potential vanishes separately. We shall now analyze the condition for vanishing of various terms separately. Our method differs from the one used in [17] in that in [17] we used gauge invariant combinations of variables for analyzing the Fterm equations, whereas here we work in a specific gauge. While for $N=1$ both approaches are equally efficient, working in a fixed gauge seems to make the analysis simpler for $N \geq 2$.

\subsection{F-term equations}

Vanishing of the F-term contribution to the potential requires that $\partial \mathcal{W} / \partial \varphi_{\alpha}$ vanishes for each $\alpha$. The $\partial \mathcal{W} / \partial \Phi_{m}^{(k)}=0$ equations give

$$
Z^{(k \ell)} Z^{(\ell k)}=-c^{(k \ell)} \quad \text { for } \quad 1 \leq k, \ell \leq 4, \quad k \neq \ell .
$$

The $\partial \mathcal{W} / \partial Z^{(k \ell)}=0$ equations give

$$
\begin{gathered}
\sum_{m=1}^{3} \varepsilon^{k \ell m}\left(Z^{(\ell k)} \Phi_{m}^{(k)}-\Phi_{m}^{(\ell)} Z^{(\ell k)}\right)+\sum_{\substack{m=1 \\
m \neq k, \ell}}^{4} Z^{(\ell m)} Z^{(m k)}(-1)^{\delta_{k 1} \delta_{\ell 3} \delta_{m 4}}=0 \\
\text { for } 1 \leq k, \ell \leq 3, \quad k \neq \ell, \\
\left(\Phi_{k}^{(k)} Z^{(k 4)}-Z^{(k 4)} \Phi_{k}^{(4)}\right)+\sum_{\substack{\ell=1 \\
\ell \neq k}}^{3} Z^{(k \ell)} Z^{(\ell 4)}(-1)^{\delta_{k 1} \delta_{\ell 3}}=0 \quad \text { for } \quad 1 \leq k \leq 3, \\
\left(Z^{(4 k)} \Phi_{k}^{(k)}-\Phi_{k}^{(4)} Z^{(4 k)}\right)+\sum_{\substack{m=1 \\
m \neq k}}^{3} Z^{(4 m)} Z^{(m k)}(-1)^{\delta_{m 1} \delta_{k 3}}=0 \text { for } 1 \leq k \leq 3 .
\end{gathered}
$$

Since the superpotential has a large group of symmetries, in order to find solutions to these F-term equations we need to choose one representative from each symmetry orbit. First we use the shift symmetries (2.11) to choose

$$
\Phi_{1}^{(1)}=0, \quad \Phi_{2}^{(1)}=0, \quad \Phi_{3}^{(1)}=0, \quad \Phi_{1}^{(2)}=0, \quad \Phi_{2}^{(2)}=0, \quad \Phi_{3}^{(3)}=0 .
$$

This effectively removes the flat directions associated with the shift symmetries. As discussed below (2.11), once we have made this choice, we can forget about the flat directions associated with these shift symmetries and also the fermionic superpartners of these flat directions. The information about $P(y)$ is contained in the rest of the system of equations.

Next we note that the superpotential is invariant under the complexified gauge transformation

$$
Z^{(k \ell)} \rightarrow a_{k}\left(a_{\ell}\right)^{-1} Z^{(k \ell)} \text { for } 1 \leq i \leq 4, \quad 1 \leq k \leq 4,
$$

where $a_{k}$ for $1 \leq k \leq 4$ are complex numbers. Therefore solutions to the F-term equations come as orbits of this symmetry group. Using this we shall now make a convenient choice of 
gauge. Of the four parameters encoded in $a_{1}, a_{2}, a_{3}$ and $a_{4}$, one combination does not act on the fields. Therefore only three are independent. Now, since $Z^{(k \ell)}$ 's for $1 \leq k, \ell \leq 4$ are complex numbers, (3.1) shows that neither $Z^{(k \ell)}$ nor $Z^{(\ell k)}$ can vanish as long as $c^{(k \ell)}$ 's are chosen to be non-zero. This allows us to fix the gauge corresponding to the transformations generated by $a_{1}, a_{3}$ and $a_{4}$ by setting

$$
Z^{(12)}=1, \quad Z^{(23)}=1, \quad Z^{(14)}=1
$$

We now substitute the gauge choices (3.3) and (3.5) into the F-term equations and look for solutions to these equations. For this we choose random rational values of the $c^{(k \ell)}$ 's for $1 \leq k, \ell \leq 4$. A numerical analysis of the Hilbert series using Singular [30] and Macaulay2 [31], treating the F-term equations as ideals of the ring, shows that the solution space is a collection of 12 points. Explicit numerical solutions in Mathematica [32] also yields precisely 12 solutions for each choice of the constants $\left\{c^{(k \ell)}\right\}$.

\subsection{D-term equations}

Next we turn to solving the D-term equations. For this we recall that the F-term equations do not give isolated solutions, but give orbits of the complexified gauge group generated by the complex numbers $a_{1}, a_{2}, a_{3}$ and $a_{4}$. The D-term equations only respect a subgroup of this symmetry group consisting of physical gauge transformations. Therefore for each of the solutions to the F-term equations we can examine the orbit under (3.4) and then try to determine $a_{1}, a_{2}, a_{3}$ and $a_{4}$ by demanding that the D-term equations are satisfied. As before, one combination of $a_{1}, a_{2}, a_{3}$ and $a_{4}$ does not act on the variables, and so we can restrict to transformations for which $a_{3}=1$. However this is not expected to fix the parameters $a_{1}, a_{2}, a_{3}$ and $a_{4}$ completely since a subgroup of the transformations (3.4), corresponding to physical gauge transformations, generates a symmetry of the D-term equations of motion as well. This subgroup is generated by taking the $a_{i}$ 's to be phases. This means that once we have found a set of $a_{i}$ 's that give a solution to the D-term equations, we can generate other solutions by performing $\mathrm{U}(1)^{4}$ transformations. The effect of these transformations will be to transform the parameters $a_{1}, a_{2}, a_{3}$ and $a_{4}$ by

$$
a_{k} \rightarrow e^{i \phi_{k}} a_{k}
$$

where $\phi_{k}$ are real numbers. Therefore for finding solutions up to gauge transformations, we can use the transformations (3.6) to fix the 'gauge' for $a_{1}, a_{2}, a_{3}$ and $a_{4}$. We choose a gauge in which all the $a_{k}$ 's are real and positive.

We now transform each of the 12 solutions to the F-term equations by (3.4) with real positive $a_{k}$ 's and try to determine the $a_{k}$ 's by solving the D-term equations. For each of the 12 cases, we find that there is a unique choice of real positive $a_{k}$ 's that solves the D-term equations. This shows that up to gauge transformations, there are precisely 12 solutions to the F- and D-term equations. More accurately we have 12 different gauge orbits, generated by $\mathrm{U}(1)^{4}$ transformation, as solutions to the F- and D-term equations. 


\section{$3.3 X_{i}^{(k)}$ dependent terms}

Although at the end of appendix A we shall give a general argument that all $X_{i}^{(k)}$ 's must vanish up to the shift symmetry described in the last line of (2.11), we shall now verify this explicitly for this example. For this we have to demand the vanishing of the $X_{i}^{(k)}$ dependent terms in the potential given in (2.9). Since vanishing of F- and D-terms is a necessary condition for a supersymmetric vacuum, the choice of $Z^{(k \ell)}$ 's and $\Phi_{i}^{(k)}$ 's must be restricted to the 12 solutions that we have found. Now we note that since (2.9) is a sum of positive definite terms, in order for $V_{\text {gauge }}$ to vanish, each term in the potential must vanish. In particular this will require

$$
\left(X_{i}^{(k)}-X_{i}^{(\ell)}\right) Z^{(k \ell)}=0, \quad \text { for } \quad 1 \leq k, \ell \leq 4, \quad k \neq \ell, \quad 1 \leq i \leq 3 .
$$

Using the result that the $Z^{(k \ell)}$ are non-zero, we get $X_{i}^{(k)}=X_{i}^{(\ell)}$. Using the shift symmetry given in the third line of $(2.11)$ we can set $X_{i}^{(1)}$ to zero for $1 \leq i \leq 3$. As a result $X_{i}^{(k)}$ for $k=2,3$ and 4 must also vanish. This shows that the only way to make the potential vanish is to have all the $X_{i}^{(k)}$ 's vanish for $1 \leq k \leq 4$ and $1 \leq i \leq 3$.

\subsection{Gauss' law constraint}

Finally we turn to the Gauss' law constraint. This is not a constraint on the classical configuration but on the quantum vacuum. When we dimensionally reduce the $3+1$ dimensional theory to $0+1$ dimensions we also get non-dynamical fields from the zeroth component of the gauge fields which we have set to zero. The equations of motion for $A_{0}^{(k)}$ imposes the constraint that the total gauge charge carried by the state must vanish. Since for a charged complex scalar the gauge charge will involve a product of the field and its time derivative, the gauge charge will vanish for a classical solution which is time independent. But we still need to examine if the quantum ground state - which can be regarded as the ground state of a system of coupled harmonic oscillators describing small oscillations around the isolated vacua, and a set of flat directions generated by the gauge transformation - is invariant under the gauge transformation.

Now as already mentioned, the solutions to the potential minimization equations generate 12 different gauge orbits. The effect of gauge transformation is to move a point in the vacuum manifold along the gauge orbit. Therefore the requirement that the quantum ground state is gauge invariant simply translates to the requirement that the ground state wave-function is independent of the coordinates along the gauge orbit. By expressing the kinetic term for each variable $\left(Z^{(k \ell)}\right.$ 's and $\Phi_{i}^{(k)}$ 's) in terms of the collective coordinates generated by the gauge transformations we can bring the Lagrangian of the collective coordinates to that of three independent free particles (corresponding to three independent gauge transformations that act non-trivially on the fields) with compact target space and positive masses. Therefore the gauge invariant state indeed is the lowest energy state of the system. This leads us to conclude that for each of the 12 gauge orbits, the quantum ground state is invariant under gauge transformations and hence satisfies the Gauss' law constraint.

Therefore we see from (2.12) that the for $N=1$ we have

$$
P(y)=12 \text {. }
$$


The $y$ independence of $P(y)$ is the result of the gauge inequivalent solutions being isolated points, and is consistent with the conjecture that all the microstates carry zero angular momentum.

\section{Supersymmetric ground states for $N=2$}

We shall now determine the number of supersymmetric ground states for $N=2$ following the same steps as in section 3 .

\subsection{F-term equations}

Vanishing of the F-term contribution to the potential requires that $\partial \mathcal{W} / \partial \varphi_{\alpha}$ vanishes for each $\alpha$. The $\partial \mathcal{W} / \partial \Phi_{m}^{(k)}=0$ equations give

$$
\begin{aligned}
& Z^{(k \ell)} Z^{(\ell k)}=-c^{(k \ell)} \quad \text { for } \quad 1 \leq k, \ell \leq 3, \quad k \neq \ell, \\
& Z^{(k 4)} Z^{(4 k)}=-2 c^{(k 4)}, \quad 1 \leq k \leq 3, \\
& Z^{(4 k)} Z^{(k 4)}=-c^{(k 4)} I_{2}-\sum_{\ell, m=1}^{3} \varepsilon^{k \ell m} \Phi_{\ell}^{(4)} \Phi_{m}^{(4)}, \quad 1 \leq k \leq 3 .
\end{aligned}
$$

The equations in the second line follow from the trace of the equation in the third line, but we have listed them separately as they will be useful in analyzing the solutions. The $\partial \mathcal{W} / \partial Z^{(k \ell)}=0$ equations give

$$
\begin{aligned}
& \sum_{m=1}^{3} \varepsilon^{k \ell m}\left(Z^{(\ell k)} \Phi_{m}^{(k)}-\Phi_{m}^{(\ell)} Z^{(\ell k)}\right)+\sum_{\substack{m=1 \\
m \neq k, \ell}}^{4} Z^{(\ell m)} Z^{(m k)}(-1)^{\delta_{k 1} \delta_{\ell 3} \delta_{m 4}}=0 \\
& \text { for } \quad 1 \leq k, \ell \leq 3, \quad k \neq \ell, \\
& \left(\Phi_{k}^{(k)} Z^{(k 4)}-Z^{(k 4)} \Phi_{k}^{(4)}\right)+\sum_{\substack{\ell=1 \\
\ell \neq k}}^{3} Z^{(k \ell)} Z^{(\ell 4)}(-1)^{\delta_{k 1} \delta_{\ell 3}}=0 \quad \text { for } \quad 1 \leq k \leq 3, \\
& \left(Z^{(4 k)} \Phi_{k}^{(k)}-\Phi_{k}^{(4)} Z^{(4 k)}\right)+\sum_{\substack{m=1 \\
m \neq k}}^{3} Z^{(4 m)} Z^{(m k)}(-1)^{\delta_{m 1} \delta_{k 3}}=0 \quad \text { for } \quad 1 \leq k \leq 3 .
\end{aligned}
$$

As in section 3, we use the shift symmetries (2.11) to choose

$$
\Phi_{1}^{(1)}=0, \quad \Phi_{2}^{(1)}=0, \quad \Phi_{3}^{(1)}=0, \quad \Phi_{1}^{(2)}=0, \quad \Phi_{2}^{(2)}=0, \quad \Phi_{3}^{(3)}=0 .
$$

Next we note that the superpotential is invariant under the complexified gauge transformation

$$
\begin{gathered}
Z^{(k \ell)} \rightarrow a_{k}\left(a_{\ell}\right)^{-1} Z^{(k \ell)}, \quad Z^{(4 k)} \rightarrow\left(a_{k}\right)^{-1} M Z^{(4 k)}, \quad Z^{(k 4)} \rightarrow a_{k} Z^{(k 4)} M^{-1}, \\
\text { for } 1 \leq k \leq 3, \quad k \neq \ell, \\
\Phi_{i}^{(k)} \rightarrow \Phi_{i}^{(k)}, \quad \Phi_{i}^{(4)} \rightarrow M \Phi_{i}^{(4)} M^{-1}, \quad \text { for } \quad 1 \leq i \leq 3, \quad 1 \leq k \leq 3,
\end{gathered}
$$

where $a_{k}$ for $1 \leq k \leq 3$ are complex numbers and $M$ is a $2 \times 2$ complex matrix. Therefore solutions to the F-term equations come as orbits of the symmetry group. Using this we 
shall now make a convenient choice of gauge. Since $Z^{(k \ell)}$ 's for $1 \leq k, \ell \leq 3$ are complex numbers the first equation in (4.1) shows that neither $Z^{(k \ell)}$ nor $Z^{(\ell k)}$ can vanish as long as $c^{(k \ell)}$ 's are chosen to be non-zero. This allows us to fix the gauge corresponding to the transformations generated by $a_{1}$ and $a_{3}$ by setting

$$
Z^{(12)}=1, \quad Z^{(23)}=1 .
$$

Similarly, since $Z^{(k 4)}$ are two component row vectors and $Z^{(4 k)}$ are two component column vectors for $1 \leq k \leq 3$, the second equation in (4.1) tells us that neither $Z^{(k 4)}$ nor $Z^{(4 k)}$ can have both components vanishing. This allows us to use the transformation generated by $M$ to set

$$
Z^{(14)}=\left(\begin{array}{ll}
1 & 0
\end{array}\right)
$$

This does not fix $M$ completely since the choice of $M$ given by

$$
\left(\begin{array}{ll}
1 & 0 \\
r & s
\end{array}\right) \text {. }
$$

preserves the form of $Z^{(14)}$. Since $Z^{(24)}$ is non-zero, we can use this residual gauge symmetry to set

$$
Z^{(24)}=\left(\begin{array}{ll}
0 & 1
\end{array}\right)
$$

There is one case where this gauge condition fails, and that is in the case when $Z^{(24)}$ is parallel to $Z^{(14)}$ to begin with. In this case the gauge symmetry described in (4.7) cannot be used to bring $Z^{(24)}$ to the form (4.8). We shall deal with this case separately. Once we have used $a_{1}, a_{3}$ and $M$ to fix these gauges, we cannot use $a_{2}$ any more since its action is determined in terms of the others.

We now substitute the gauge choices (4.3), (4.5), (4.6) and (4.8) into the F-term equations and look for solutions to these equations. For this we choose random rational values of the $c^{(k \ell)}$ 's for $1 \leq k<\ell \leq 4$. A numerical analysis of the Hilbert series using Singular and Macaulay2, treating the F-term equations as ideals of the ring, shows that the solution space is a collection of 56 points. Explicit numerical solutions in Mathematica also yields precisely 56 solutions for each choice of the constants $\left\{c^{(k \ell)}\right\}$.

We also explore the possibility of having solutions with $Z^{(24)}$ proportional to $Z^{(14)}$ for which the gauge choice (4.8) will be invalid. In this case the analysis of the Hilbert series shows that there are no solutions. Explicit attempts to find solutions to the F-term equations in Mathematica also gives no results. This shows that there are no solutions for which the gauge choice (4.8) breaks down.

\section{$4.2 \quad$ D-term equations}

The solutions to the F-term equations give orbits of the complexified gauge group generated by the complex numbers $a_{1}, a_{2}, a_{3}$ and the $2 \times 2$ complex matrix $M$ as given in (4.4). The D-term equations only respect a subgroup of this symmetry group consisting of physical gauge transformations. Therefore for each of the solutions to the F-term equations we can examine the orbit under (4.4) and then try to determine $a_{1}, a_{2}, a_{3}$ and $M$ by demanding 
that the D-term equations are satisfied. As before, one combination of $a_{1}, a_{2}, a_{3}$ and $\operatorname{det} M$ do not act on the variables, and so we can restrict to transformations for which $\operatorname{det} M=1$. However this is not expected to fix the parameters $a_{1}, a_{2}, a_{3}$ and $M$ completely since a subgroup of the transformations (4.4), corresponding to physical gauge transformations, generates a symmetry of the D-term equations of motion as well. This subgroup is generated by taking the $a_{i}$ to be phases and $M$ to be an $\mathrm{SU}(2)$ matrix. This means that once we have found a set of $a_{i}$ 's and $M$ that give a solution to the D-term equations, we can generate other solutions by performing $\mathrm{U}(1)^{3} \times \mathrm{SU}(2)$ transformations. The effect of these transformations will be to transform the parameters $a_{1}, a_{2}, a_{3}$ and $M$ by

$$
a_{k} \rightarrow e^{i \phi_{k}} a_{k}, \quad M \rightarrow U M,
$$

where the $\phi_{k}$ are real numbers and $U$ is an $\mathrm{SU}(2)$ matrix. Therefore for finding solutions up to gauge transformations, we can use the transformations (4.9) to fix the 'gauge' for $a_{1}$, $a_{2}, a_{3}$ and $M$. Using the transformations generated by the $\phi_{k}$ 's we can make the $a_{k}$ 's real and positive. Furthermore, one can easily check that using the transformations generated by $U$ and the constraint $\operatorname{det} M=1$, we can bring $M$ to the form

$$
M=\left(\begin{array}{cc}
1 / a & b \\
0 & a
\end{array}\right),
$$

where $a$ is a real positive number and $b$ is a complex number.

We now transform each of the 56 solutions to the F-term equations by (4.4) with real positive $a_{k}$ 's and $M$ of the form (4.10) and try to determine the $a_{k}$ 's, $a$ and $b$ by numerically solving the D-term equations. For each of the 56 cases, we find that there is a unique choice of real positive $a_{k}$ 's and $M$ of the form (4.10) that solves the D-term equations. This shows that up to gauge transformations, there are precisely 56 solutions to the $\mathrm{F}$ - and D-term equations. More accurately we have 56 different gauge orbits, generated by $\mathrm{U}(1)^{3} \times \mathrm{SU}(2)$ transformation, as solutions to the F- and D-term equations.

\section{3 $X_{i}^{(k)}$ dependent terms}

Next we have to check if the $X_{i}^{(k)}$ dependent terms in the potential given in (2.9) vanish. For each of the 56 solutions there is a simple way to make these terms vanish - we simply choose $X_{i}^{(k)}=0$ for $1 \leq i \leq 3,1 \leq k \leq 4$. The question we shall be interested in is: are there other configurations that make the $X_{i}^{(k)}$ dependent terms vanish?

Since vanishing of F- and D-terms is a necessary condition for a supersymmetric vacuum, the choice of $Z^{(k \ell)}$ 's and $\Phi_{i}^{(k)}$ 's must be restricted to the 56 solutions that we have found. We shall now argue that for each of these solutions, the $X_{i}^{(k)}$ 's for $1 \leq k \leq 4$ and $1 \leq i \leq 3$ must vanish identically up to the shift symmetries described in the last line of (2.11). For this we turn to the potential (2.9) and note that since this is a sum of positive definite terms, in order for $V_{\text {gauge }}$ to vanish, each term in the potential must vanish. In particular this will require

$$
X_{i}^{(k)} Z^{(k \ell)}-Z^{(k \ell)} X_{i}^{(\ell)}=0, \quad \text { for } \quad 1 \leq k, \ell \leq 4, \quad k \neq \ell, \quad 1 \leq i \leq 3 .
$$


For $1 \leq k, \ell \leq 3, k \neq \ell, X_{i}^{(k)}$ and $Z^{(k \ell)}$ are numbers and hence, using the result that the $Z^{(k \ell)}$ are non-zero, we get $X_{i}^{(k)}=X_{i}^{(\ell)}$. Using the shift symmetry given in the third line of (2.11) we can set $X_{i}^{(1)}$ to zero for $1 \leq i \leq 3$. As a result $X_{i}^{(2)}$ and $X_{i}^{(3)}$ must also vanish. Choosing $k=4$ and $\ell=1,2$ or 3 in (4.11), and vice versa, we now get

$$
X_{i}^{(4)} Z^{(4 \ell)}=0, \quad Z^{(\ell 4)} X_{i}^{(4)}=0 .
$$

Since we have seen that $Z^{(4 \ell)}$ and $Z^{(\ell 4)}$ cannot have their both components vanish, this shows that $Z^{(4 \ell)}$ and $Z^{(\ell 4)}$ are right and left eigenvectors of $X_{i}^{(4)}$ with zero eigenvalues. Let us suppose that $X_{i}^{(4)}$ is non-zero for at least one $i$. In that case the $Z^{(\ell 4)}$ 's for $1 \leq \ell \leq 3$ must be proportional to each other since each of them is a left eigenvector of the nonvanishing $X_{i}^{(4)}$ with zero eigenvalue. However as already mentioned, while examining the validity of the gauge choice (4.8) we have explicitly checked that there are no solutions to the F-term equations in which $Z^{(14)}$ and $Z^{(24)}$ are parallel to each other. This shows that our initial assumption must have been wrong, and $X_{i}^{(4)}$ for each $i$ must vanish identically. This shows that the only way to make the potential vanish is to have all the $X_{i}^{(k)}$ 's vanish for $1 \leq k \leq 4$ and $1 \leq i \leq 3$.

\subsection{Gauss' law constraint}

Finally we turn to the Gauss' law constraint. This analysis is identical to that given in section 3.4 except for one difference: the collective modes associated with the $\mathrm{SU}(2)$ gauge transformations have a kinetic term given by that of a rigid rotator with positive definite inertia matrix instead of that of a free particle with positive mass. Positive definiteness of the inertia matrix guarantees that the ground state wave-function is independent of these collective coordinates and hence the ground state is gauge invariant. Thus the Gauss' law constraint is automatically satisfied for each of the 56 gauge orbits.

Therefore we see from (2.12) that for $N=2$ we have

$$
P(y)=56 .
$$

As in section 3, the $y$ independence of $P(y)$ is the result of the gauge inequivalent solutions being isolated points, and is consistent with the conjecture that all the microstates carry zero angular momentum. We also see from (2.14) and (2.15) that the counting in the dual description gives a BPS index 56. Thus there is perfect agreement between our result and that in the dual description.

\section{Supersymmetric ground states for $N=3$}

The analysis of the equations for the $N=3$ case proceeds similarly to that for the $N=2$ case. Up to (4.5) there is essentially no change. The gauge conditions (4.6) and (4.8) are replaced by

$$
Z^{(14)}=(1,0,0), \quad Z^{(24)}=(0,1,0), \quad Z^{(34)}=(0,0,1) .
$$

Such a gauge choice is always possible if initially the vectors $Z^{(14)}, Z^{(24)}$ and $Z^{(34)}$ are linearly independent. The case where they are linearly dependent is analyzed separately 
and we find no solution in this sector. With the gauge choice (5.1) we find 208 distinct solutions to the set of F-term constraints. Furthermore for each of these solutions the $X_{i}^{(k)}$ 's can be shown to vanish identically using arguments identical to those given below (4.12). We have not checked explicitly that for each of these solutions we have a unique solution to the D-term constraints up to gauge transformation, but since the D-term constraints usually amount to quotienting by complexified gauge transformations, and since following arguments similar to the one given below (4.5) one can argue that none of the vectors $Z^{(4 k)}$ and $Z^{(k 4)}$ can vanish as a vector, one expects on general grounds that the quotient by complexified gauge transformation will give a unique solution for each solution to the F-term constraints. Therefore we conclude that in this case we have

$$
P(y)=208 \text {. }
$$

Again the $y$ independence of $P(y)$ is the result of the gauge inequivalent solutions being isolated points, and is consistent with the conjecture that all the microstates carry zero angular momentum. (5.2) is in perfect agreement with the result in the dual description which, according to (2.14) and (2.15), gives $P(-1)=208$.

\section{Conclusion}

In this paper we have provided evidence for the conjecture that all the microstates of single centered BPS black holes carry strictly zero momentum at a generic point in the moduli space. This conjecture is consistent with the near horizon $A d S_{2} \times S^{2}$ geometry of extremal black holes, but there are no direct arguments in the microscopic theory leading to this conjecture. Therefore the test of this conjecture provides evidence that the black hole horizon carries more information than just some average properties of the microstates.

These results put a strong constraint on possible fuzzball solutions describing black hole microstates [33-37]. Typical fuzzball solutions are constructed at special points in the moduli space and carry states of different angular momenta. For demonstrating that they describe genuine black hole microstates, one needs to construct these solutions for generic values of the asymptotic moduli and show that the solutions so obtained carry strictly zero angular momentum.

A different approach to this problem has been suggested in [38] where one constructs solutions with asymptotic $A d S_{2}$ boundary conditions. These solutions carry strictly zero angular momentum. However these are not truly in the spirit of the fuzzball program since they exist within the near horizon geometry of the black hole instead of replacing the near horizon geometry by a smooth solution. Furthermore since these solutions have two asymptotic boundaries, they most likely describe an entangled state living on two copies of the black hole Hilbert space instead of the microstates of a single black hole [39].

\section{Acknowledgments}

We wish to thank Atish Dabholkar, Joao Gomes, Dileep Jatkar, Jan Manschot, Noppadol Mekareeya, Sameer Murthy, Boris Pioline and Savdeep Sethi for useful discussions. We 
have benefited from the use of symbolic manipulation programs Macaulay2, Mathematica and Singular, and the use of the High Performance Cluster Computing facility at the Harish-Chandra Research Institute. This work was supported in part by the DAE project 12-R\&D-HRI-5.02-0303. The work of A.S. was also supported in part by the J. C. Bose fellowship of the Department of Science and Technology, India.

\section{A Normalization of $Z-Z-Z$ coupling}

In this appendix we shall determine the normalizations and signs of the $Z-Z-Z$ coupling appearing in (2.2) by analyzing respectively open string amplitudes and symmetry requirements. The computation will be done around a background in which all the circles of $T^{6}$ are orthonormal and have radius $\sqrt{\alpha^{\prime}}$. Fluctuations away from this background will be parametrized by the constants $c^{(k \ell)}$ and $c^{(k)}$ appearing in (2.3) and (2.7). Since we work in the region where these constants are small (see footnote 6), the corrections to the cubic terms in the superpotential proportional to these constants can be ignored.

The easiest way to determine a cubic term in the superpotential is to examine the Yukawa coupling between two fermions and one boson that arises from this term. For this we need to construct the vertex operators of the corresponding states and compute their three point function on the disk. We shall denote by $b$ and $c$ the usual diffeomorphism ghost fields, by $\beta$ and $\gamma$ the superconformal ghosts and by $\phi$ the scalar that arises from bosonization of the $\beta-\gamma$ system [40], normalized such that

$$
\left\langle c\left(z_{1}\right) e^{-\phi}\left(z_{1}\right) c\left(z_{2}\right) e^{-\phi / 2}\left(z_{2}\right) c\left(z_{3}\right) e^{-\phi / 2}\left(z_{3}\right)\right\rangle=\left(z_{1}-z_{2}\right)^{1 / 2}\left(z_{1}-z_{3}\right)^{1 / 2}\left(z_{2}-z_{3}\right)^{3 / 4},
$$

up to a sign. In the matter sector, we shall combine the compact spatial coordinates into complex coordinates as

$$
w^{1}=x^{4}+i x^{5}, \quad w^{2}=x^{6}+i x^{7}, \quad w^{3}=x^{8}+i x^{9} .
$$

$w^{1}, \ldots, w^{3}$ are complex coordinates. For each coordinate $w^{i}$ we have a complex worldsheet scalar field which we shall denote by $W^{i}$. Their superpartners are complex world sheet fermions which we denote by $\psi^{i}$. We also introduce the complex spin field $s_{i}$ that twists $\psi^{i}$ by a $Z_{2}$ transformation $\psi^{i} \rightarrow-\psi^{i}$ and the real twist field $\sigma_{i}$ that twists $W^{i}$ by a $Z_{2}$ transformation $W^{i} \rightarrow-W^{i}$. Since a world-sheet scalar satisfying a Neumann boundary condition at one end and a Dirichlet boundary condition at the other end has a half-integer mode expansion, the twist fields $\sigma_{i}$ will be necessary for constructing the vertex operators for open string states satisfying Neumann-Dirichlet boundary conditions in some directions. The spin fields $s_{i}$ will be needed for constructing the vertex operators for open string states satisfying Neumann-Dirichlet boundary conditions in some directions and also for constructing vertex operators in the Ramond sector. Finally we shall denote by $s_{\alpha}^{(n c)}$ the spin fields associated with the non-compact directions carrying spinor index $\alpha$ of $\mathrm{SO}(3,1)$. We shall use standard normalizations for the fields $\psi^{i}, s_{i}$ and $\sigma_{i}$, e.g.

$$
\psi^{i}\left(z_{1}\right) \bar{\psi}^{j}\left(z_{2}\right)=\delta_{i j}\left(z_{1}-z_{2}\right)^{-1}, \quad s_{i}\left(z_{1}\right) \bar{s}_{j}\left(z_{2}\right)=\delta_{i j}\left(z_{1}-z_{2}\right)^{-1 / 4},
$$




$$
\begin{aligned}
\bar{\psi}^{i}\left(z_{1}\right) s_{j}\left(z_{2}\right) & =\delta_{i j}\left(z_{1}-z_{2}\right)^{-1 / 2} \bar{s}_{j}\left(z_{2}\right), & \psi^{i}\left(z_{1}\right) \bar{s}_{j}\left(z_{2}\right) & =\delta_{i j}\left(z_{1}-z_{2}\right)^{-1 / 2} s_{j}\left(z_{2}\right), \\
\sigma_{i}\left(z_{1}\right) \sigma_{j}\left(z_{2}\right) & =\delta_{i j}\left(z_{1}-z_{2}\right)^{-1 / 4}, & s_{\alpha}^{(n c)}\left(z_{1}\right) s_{\beta}^{(n c)}\left(z_{2}\right) & =\epsilon_{\alpha \beta}\left(z_{1}-z_{2}\right)^{-1 / 2},
\end{aligned}
$$

up to multiplicative signs and less singular additive terms. The operator products given in the last line are determined by the conformal weights of $\sigma_{i}, s_{\alpha}^{(n c)}$ and their normalizations. The operator products in the first two lines can be shown to be mutually compatible by bosonizing the fermions $\psi^{i}$ to scalar fields $\phi_{i}$ and using the identification

$$
\psi^{i}=e^{i \phi_{i}}, \quad \bar{\psi}^{i}=e^{-i \phi_{i}}, \quad s_{i}=e^{i \phi_{i} / 2}, \quad \bar{s}_{i}=e^{-i \phi_{i} / 2} .
$$

The other ingredient we need for the construction of the vertex operator is Chan-Paton factors. Since we have altogether $N+3$ D-branes, the Chan-Paton factors can be taken to be $(N+3) \times(N+3)$ matrices. We shall choose the convention in which the first three rows and columns represent the branes 1, 2 and 3 and the last $N$ rows and columns label the brane stack 4 . In this notation, for $1 \leq k, \ell \leq 3,1 \leq r, s \leq N$, the Chan-Paton factors for the vertex operators for $\Phi_{\ell}^{(k)},\left(\Phi_{\ell}^{(4)}\right)_{r s}, Z^{(k \ell)}, Z_{r}^{(k 4)}$ and $Z_{r}^{(4 k)}$ will be matrices whose only non-zero entries are the $(k, k)^{\prime}$ th, $(3+r, 3+s)^{\prime}$ 'th, $(k, \ell)^{\prime}$ th, $(k, 3+r)^{\prime}$ 'th and $(3+r, k)^{\prime}$ 'th elements, respectively. In the analysis that follows we shall suppress the ChanPaton factors - they just accompany the vertex operators as multiplicative matrices. The correlation function of a given set of vertex operators will contain a term proportional to the trace of the ordered product of their Chan-Paton factors.

The vertex operators for the states corresponding to $\Phi_{i}^{(k)}$ and $Z^{(k \ell)}$ can be constructed using these operators. For a superfield $A$ we shall denote by $V_{A, \alpha}^{f}$ and $V_{A}^{b}$ the vertex operators of the fermionic and bosonic components of the superfield. Here $\alpha$ denotes a $3+1$ dimensional spinor index. In this notation we have, for example

$$
\begin{aligned}
V_{\Phi_{1}^{(k)}}^{b} & =c e^{-\phi} \psi^{1}, & V_{\Phi_{1}^{(k)}, \alpha}^{f} & =c e^{-\phi / 2} s_{1} \bar{s}_{2} \bar{s}_{3} s_{\alpha}^{(n c)}, \\
V_{Z^{(12)}}^{b} & =c e^{-\phi} \sigma_{1} \sigma_{2} s_{1} s_{2}, & V_{Z^{(12)}, \alpha}^{f} & =c e^{-\phi / 2} \sigma_{1} \sigma_{2} \bar{s}_{3} s_{\alpha}^{(n c)},
\end{aligned}
$$

up to multiplicative signs. Here the $\psi^{1}$ factor in the expression for $V_{\Phi_{1}^{(k)}}^{b}$ reflects that this mode represents position / Wilson line on the brane along $w^{1}$, whereas the $s_{1} \bar{s}_{2} \bar{s}_{3}$ factor in $V_{\Phi_{1}^{(k)}, \alpha}^{f}$ is obtained by starting with the operator $\bar{s}_{1} \bar{s}_{2} \bar{s}_{3}$ that appears in the expression for the supersymmetry generator, and taking the leading term in its operator product with $\psi^{1}$ - the same factor that is present in $V_{\Phi_{1}^{(k)}}^{b}$. In the expression for $V_{Z^{(12)}}^{b}$ the $\sigma_{1} \sigma_{2}$ and $s_{1} s_{2}$ factors reflect that the fields $W^{1}$ and $W^{2}$ and their fermionic partners $\psi^{1}$ and $\psi^{2}$ satisfy Neumann boundary conditions at one end of the open string and Dirichlet boundary conditions at the other end. On the other hand the $\sigma_{1} \sigma_{2} \bar{s}_{3}$ term in the expression for $V_{Z^{(12)}, \alpha}^{f}$ is the leading term in the expression for the operator product of $\bar{s}_{1} \bar{s}_{2} \bar{s}_{3}$ and $\sigma_{1} \sigma_{2} s_{1} s_{2}$. Following this strategy we can find the expressions for all the vertex operators $V_{\Phi_{i}^{(k)}}^{b}, V_{\Phi_{i}^{(k)}, \alpha}^{f}, V_{Z^{(k \ell)}}^{b}$ and $V_{Z^{(k \ell)}, \alpha}^{f}$.

Let us now calculate the three point function

$$
\left\langle V_{\Phi_{1}^{(4)}}^{b} V_{\Phi_{2}^{(4)}, \alpha}^{f} V_{\Phi_{3}^{(4)}, \beta}^{f}\right\rangle,
$$


to fix the normalization of the terms in $\mathcal{W}_{4}$ in (2.4). Using the generalization of (A.5) we see that this is computed using the correlation function

$$
\left\langle c e^{-\phi} \psi^{1}\left(z_{1}\right) c e^{-\phi / 2} \bar{s}_{1} s_{2} \bar{s}_{3} s_{\alpha}^{(n c)}\left(z_{2}\right) c e^{-\phi / 2} \bar{s}_{1} \bar{s}_{2} s_{3} s_{\beta}^{(n c)}\left(z_{3}\right)\right\rangle .
$$

This correlator can be factorized and evaluated using (A.1) and (A.3) as

$$
\begin{aligned}
& \left\langle c e^{-\phi}\left(z_{1}\right) c e^{-\phi / 2}\left(z_{2}\right) c e^{-\phi / 2}\left(z_{3}\right)\right\rangle_{\text {ghost }}\left\langle\psi^{1}\left(z_{1}\right) \bar{s}_{1}\left(z_{2}\right) \bar{s}_{1}\left(z_{3}\right)\right\rangle_{\psi^{1}} \\
& \left\langle s_{2}\left(z_{2}\right) \bar{s}_{2}\left(z_{3}\right)\right\rangle_{\psi^{2}}\left\langle\bar{s}_{3}\left(z_{2}\right) s_{3}\left(z_{3}\right)\right\rangle_{\psi^{3}}\left\langle s_{\alpha}^{(n c)}\left(z_{2}\right) s_{\beta}^{(n c)}\left(z_{3}\right)\right\rangle_{n c} \\
& \quad=\epsilon_{\alpha \beta}
\end{aligned}
$$

up to a sign. This agrees with the normalization of the three point coupling of $\Phi^{(4)}$ given in (2.4) after ignoring the overall $\sqrt{2}$ factor. (Since the $\sqrt{2}$ factor appears universally in front of all terms in the superpotential $\mathcal{W}$, we can ignore this for fixing the relative normalization between different terms.)

Next we compute the $\Phi-Z-Z$ three point function. For example the $\Phi_{3}^{(1)} Z^{(12)} Z^{(21)}$ coupling will be given by the coefficient of $\epsilon_{\alpha \beta}$ in

$$
\begin{aligned}
\left\langle V_{\Phi_{3}^{(1)}}^{b}\left(z_{1}\right) V_{Z^{(12)}, \alpha}^{f}\left(z_{2}\right) V_{Z^{(21)}, \beta}^{f}\left(z_{3}\right)\right\rangle & =\left\langle c e^{-\phi} \psi^{3}\left(z_{1}\right) c e^{-\phi / 2} \sigma_{1} \sigma_{2} \bar{s}_{3} s_{\alpha}^{(n c)}\left(z_{2}\right) c e^{-\phi / 2} \sigma_{1} \sigma_{2} \bar{s}_{3} s_{\beta}^{(n c)}\left(z_{3}\right)\right\rangle \\
& =\epsilon_{\alpha \beta}
\end{aligned}
$$

up to a sign. In the last step we have used (A.1) and (A.3) and factorized the correlator into contributions from the ghost sector and different components of the matter sector. This is in agreement with the normalization of the $\Phi-Z-Z$ coupling given in (2.1).

Finally let us compute the $Z-Z-Z$ three point coupling. For definiteness we focus on the $Z^{(12)} Z^{(23)} Z^{(31)}$ coupling. For this we compute

$$
\begin{aligned}
& \left\langle V_{Z^{(12)}}^{b}\left(z_{1}\right) V_{Z^{(23), \alpha}}^{f}\left(z_{2}\right) V_{Z^{(31)}, \beta}^{f}\left(z_{3}\right)\right\rangle \\
& =\left\langle c e^{-\phi} \sigma_{1} \sigma_{2} s_{1} s_{2}\left(z_{1}\right) c e^{-\phi / 2} \sigma_{2} \sigma_{3} \bar{s}_{1} s_{\alpha}^{(n c)}\left(z_{2}\right) c e^{-\phi / 2} \sigma_{3} \sigma_{1} \bar{s}_{2} s_{\beta}^{(n c)}\left(z_{3}\right)\right\rangle \\
& =\epsilon_{\alpha \beta}
\end{aligned}
$$

Again in the last step we have used (A.1) and (A.3) and factorized the correlator into contributions from the ghost sector and different components of the matter sector. The result is valid only up to a sign. This agrees with the normalization of the $Z$ - $Z$ - $Z$ coupling in $\mathcal{W}_{2}$ given in $(2.2)$.

Following the same procedure we can show that the coefficients of all the $\Phi-\Phi-\Phi, \Phi-Z$ $Z$ and $Z-Z-Z$ couplings are unity up to signs. In principle these signs can be determined by careful string theory computation but we shall describe an alternative approach based on symmetry considerations. For this we consider a more general system than what has been discussed so far, containing $N_{1}$ D2-branes along 4-5 directions, $N_{2}$ D2-branes along 6-7 directions, $N_{3}$ D2-branes along 8-9 directions and $N_{4}$ D6-branes along 4-5-6-7-8-9 directions. We claim that the correct form of the superpotential, up to field redefinition, is given by

$$
\mathcal{W}_{1}=\sqrt{2} \operatorname{Tr}\left[\left(\Phi_{3}^{(1)} Z^{(12)} Z^{(21)}-\Phi_{3}^{(2)} Z^{(21)} Z^{(12)}\right)+\left(\Phi_{1}^{(2)} Z^{(23)} Z^{(32)}-\Phi_{1}^{(3)} Z^{(32)} Z^{(23)}\right)\right.
$$




$$
\begin{aligned}
& +\left(\Phi_{2}^{(3)} Z^{(31)} Z^{(13)}-\Phi_{2}^{(1)} Z^{(13)} Z^{(31)}\right)+\left(\Phi_{1}^{(1)} Z^{(14)} Z^{(41)}-\Phi_{1}^{(4)} Z^{(41)} Z^{(14)}\right) \\
& \left.+\left(\Phi_{2}^{(2)} Z^{(24)} Z^{(42)}-\Phi_{2}^{(4)} Z^{(42)} Z^{(24)}\right)+\left(\Phi_{3}^{(3)} Z^{(34)} Z^{(43)}-\Phi_{3}^{(4)} Z^{(43)} Z^{(34)}\right)\right], \\
\mathcal{W}_{2}= & \sqrt{2} \operatorname{Tr}\left[Z^{(31)} Z^{(12)} Z^{(23)}+Z^{(13)} Z^{(32)} Z^{(21)}+Z^{(12)} Z^{(24)} Z^{(41)}+Z^{(42)} Z^{(21)} Z^{(14)}\right. \\
& \left.-Z^{(13)} Z^{(34)} Z^{(41)}+Z^{(31)} Z^{(14)} Z^{(43)}+Z^{(34)} Z^{(42)} Z^{(23)}+Z^{(43)} Z^{(32)} Z^{(24)}\right], \\
\mathcal{W}_{3}= & \sqrt{2} \operatorname{Tr}\left[c^{(12)}\left(\Phi_{3}^{(1)} \otimes I_{N_{2}}-I_{N_{1}} \otimes \Phi_{3}^{(2)}\right)+c^{(23)}\left(\Phi_{1}^{(2)} \otimes I_{N_{3}}-I_{N_{2}} \otimes \Phi_{1}^{(3)}\right)\right. \\
& +c^{(13)}\left(\Phi_{2}^{(3)} \otimes I_{N_{1}}-I_{N_{3}} \otimes \Phi_{2}^{(1)}\right)+c^{(14)}\left(\Phi_{1}^{(1)} \otimes I_{N_{4}}-I_{N_{1}} \otimes \Phi_{1}^{(4)}\right) \\
& \left.+c^{(24)}\left(\Phi_{2}^{(2)} \otimes I_{N_{4}}-I_{N_{2}} \otimes \Phi_{2}^{(4)}\right)+c^{(34)}\left(\Phi_{3}^{(3)} \otimes I_{N_{4}}-I_{N_{3}} \otimes \Phi_{3}^{(4)}\right)\right]
\end{aligned}
$$

and

$$
\begin{aligned}
\mathcal{W}_{4}= & -\sqrt{2}\left[\operatorname{Tr}\left(\Phi_{1}^{(1)} \Phi_{2}^{(1)} \Phi_{3}^{(1)}-\Phi_{1}^{(1)} \Phi_{3}^{(1)} \Phi_{2}^{(1)}\right)-\operatorname{Tr}\left(\Phi_{1}^{(2)} \Phi_{2}^{(2)} \Phi_{3}^{(2)}-\Phi_{1}^{(2)} \Phi_{3}^{(2)} \Phi_{2}^{(2)}\right)\right. \\
& \left.+\operatorname{Tr}\left(\Phi_{1}^{(3)} \Phi_{2}^{(3)} \Phi_{3}^{(3)}-\Phi_{1}^{(3)} \Phi_{3}^{(3)} \Phi_{2}^{(3)}\right)+\operatorname{Tr}\left(\Phi_{1}^{(4)} \Phi_{2}^{(4)} \Phi_{3}^{(4)}-\Phi_{1}^{(4)} \Phi_{3}^{(4)} \Phi_{2}^{(4)}\right)\right]
\end{aligned}
$$

This superpotential reduces to the one given in (2.1)-(2.4) for $N_{1}=N_{2}=N_{3}=1$. We shall now describe the arguments leading to (A.11)-(A.14).

Let us begin with the arguments leading to the form of $\mathcal{W}_{2}$. Since by field redefinitions involving changes of signs of the $Z^{(k \ell)}$ 's we can change the relative signs of various terms in $\mathcal{W}_{2}$, we only have to show that $\mathcal{W}_{2}$ is given by (A.12) up to these field redefinitions. Now these field redefinitions can only change the signs of an even number of terms in $\mathcal{W}_{2}$ since each $Z^{(k \ell)}$ appears as a factor in two of the terms. Thus an expression for $\mathcal{W}_{2}$ with an even number of minus signs cannot be turned into an expression with an odd number of minus signs and vice versa. Furthermore, it can be shown by inspection that by these field redefinitions all possible choices of $\mathcal{W}_{2}$ with an even number of minus signs can be brought to the form in which each term in $\mathcal{W}_{2}$ has positive sign, and all possible choices of $\mathcal{W}_{2}$ with an odd number of minus signs can be brought to the form given in (A.12). Thus the possible candidates for $\mathcal{W}_{2}$ can be restricted to either (A.12) or the one with all positive signs. We shall argue shortly that requiring symmetry under the exchange of different stacks of D-branes leads to the form given in (A.12).

Next we turn to $\mathcal{W}_{1}$ and $\mathcal{W}_{3}$. The shift symmetries (2.11) fix the relative sign between the pair of terms inside each parenthesis in (A.11) and (A.13), but do not fix the signs that appear in front of the parentheses. However starting with any arbitrary choice of these signs, we can arrive at (A.11) and (A.13) by redefinition involving changes of the signs of the fields $\Phi_{i}^{(k)}$ and the parameters $c^{(k \ell)}$. These field redefinitions change the signs of various terms in $\mathcal{W}_{4}$, but leave $\mathcal{W}_{2}$ unchanged.

Finally turning to $\mathcal{W}_{4}$ we see that the relative sign between the pair of terms inside each parenthesis is fixed by the requirement that these come from the dimensional reduction of $\mathcal{N}=4$ supersymmetric theories in $3+1$ dimensions. We shall see shortly that the relative 
signs between the different parentheses are fixed by the symmetry under the exchange of brane stacks. This however does not fix the overall sign of $\mathcal{W}_{4}$ leaving behind a 2 -fold ambiguity. We expect that a careful string theory calculation will be able to resolve this ambiguity, but we have not done this. As we have described in the text, the choice of $\mathcal{W}_{4}$ given in (A.14), after restriction to the case $N_{1}=N_{2}=N_{3}=1, N_{4}=N$ gives the results 12,56 and 208 for the index for $N=1,2$ and 3, respectively, in agreement with the results in the dual description. In contrast the opposite choice of sign gives the results 12, 60 and 232 for the index for the cases $N=1,2$ and 3, respectively. These do not agree with the results computed using the dual description.

What remains is to show how the exchange symmetry constrains the form of $\mathcal{W}_{2}$ and $\mathcal{W}_{4}$. For this we need to examine how the exchange symmetry acts on the coefficients $c^{(k \ell)}$. It was shown in [17] that the coefficients $c^{(k \ell)}=c^{(\ell k)}$ for $1 \leq k<\ell \leq 4$ are determined in terms of the background values of the metric and 2-form fields. For general values of $N_{1}$, $N_{2}, N_{3}$ and $N_{4}$ the results are as follows: ${ }^{10}$

$$
\begin{aligned}
& g_{47}+g_{56}=N_{12} c_{R}^{(12)}, \quad g_{57}-g_{46}=N_{12} c_{I}^{(12)}, \quad g_{49}+g_{58}=N_{13} c_{R}^{(13)}, \quad g_{59}-g_{48}=N_{13} c_{I}^{(13)}, \\
& b_{68}-b_{79}=N_{14} c_{R}^{(14)}, \quad b_{69}+b_{78}=N_{14} c_{I}^{(14)}, \quad g_{69}+g_{78}=N_{23} c_{R}^{(23)}, \quad g_{79}-g_{68}=N_{23} c_{I}^{(23)} \text {, } \\
& b_{48}-b_{59}=N_{24} c_{R}^{(24)}, \quad b_{49}+b_{58}=N_{24} c_{I}^{(24)}, \quad b_{46}-b_{57}=N_{34} c_{R}^{(34)}, \quad b_{47}+b_{56}=N_{34} c_{I}^{(34)} \text {, }
\end{aligned}
$$

where $N_{i j}=2\left(N_{i}+N_{j}\right)$ and the subscripts $R$ and $I$ stand for real and imaginary parts respectively. (A.15) generalizes the result of [17] for $N_{1}=N_{2}=N_{3}=N_{4}=1$ following the same logic. For completeness we also give the expressions for the Fayet-Iliopoulos parameters $c^{(k)}$ for $1 \leq k \leq 4$ in terms of the background fields:

$$
\begin{array}{rlrl}
c^{(1)} & =\frac{1}{2}\left(b_{45}-b_{67}-b_{89}\right)+c_{0}, & c^{(2)}=\frac{1}{2}\left(b_{67}-b_{45}-b_{89}\right)+c_{0}, \\
c^{(3)}=\frac{1}{2}\left(b_{89}-b_{45}-b_{67}\right)+c_{0}, & c^{(4)}=\frac{1}{2}\left(b_{45}+b_{67}+b_{89}\right)+c_{0},
\end{array}
$$

where $c_{0}$ is a constant that is chosen to ensure that $\sum_{k=1}^{4} c^{(k)} N_{k}=0$.

Now type IIA string theory on $T^{6}$ has an exchange symmetry $x^{4} \leftrightarrow x^{6}, x^{5} \leftrightarrow x^{7}$, $N_{1} \leftrightarrow N_{2}$ under which the D2-brane stacks 1 and 2 get exchanged and the stacks 3 and 4 remain unchanged. We see from (A.15) that under this transformation $c^{(34)}$ changes sign, $c^{(12)}$ remains unchanged, and $c^{(1 i)}$ and $c^{(2 i)}$ get exchanged for $i=3$, 4. Thus there must be an action on the variables $\Phi_{i}^{(k)}$ and $Z^{(k \ell)}$ which, together with these transformations on the $c^{(k \ell)}$ 's and $N_{i}$ 's, transform the $\mathcal{W}_{i}$ 's at most by an overall multiplicative phase. ${ }^{11}$ It is easy to verify that for the superpotential given in (A.11)-(A.14) the following accompanying transformation takes $\mathcal{W}_{i} \rightarrow-\mathcal{W}_{i}$ for $1 \leq i \leq 4$ :

$$
\left(\Phi_{3}^{(4)}, \Phi_{3}^{(3)}\right) \rightarrow\left(\Phi_{3}^{(4)}, \Phi_{3}^{(3)}\right), \quad\left(\Phi_{3}^{(2)}, \Phi_{3}^{(1)}\right) \rightarrow\left(\Phi_{3}^{(1)}, \Phi_{3}^{(2)}\right),
$$

\footnotetext{
${ }^{10}$ In [17] only the values of $\left|c^{(k \ell)}\right|$ were determined. Here we have chosen an extra minus sign in the expressions for $c_{I}^{(k \ell)}$ for $1 \leq k<\ell \leq 3$ in order to have simple realization of different symmetries.

${ }^{11}$ Multiplying the superpotential by an overall phase leaves the potential invariant.
} 


$$
\begin{aligned}
\left(\Phi_{1}^{(2)}, \Phi_{1}^{(3)}\right) & \leftrightarrow\left(\Phi_{2}^{(1)}, \Phi_{2}^{(3)}\right),\left(\Phi_{1}^{(4)}, \Phi_{1}^{(1)}\right) & \leftrightarrow-\left(\Phi_{2}^{(4)}, \Phi_{2}^{(2)}\right), & \\
Z^{(34)} & \rightarrow Z^{(34)}, & Z^{(i 1)} & \leftrightarrow-Z^{(i 2)}, \\
Z^{(12)} & \leftrightarrow-Z^{(21)}, & Z^{(43)} & \rightarrow-Z^{(43)} .
\end{aligned}
$$

On the other hand, for the choice of $\mathcal{W}_{2}$ without the sign $(-1)^{\delta_{k 1} \delta_{\ell 3} \delta_{m 4}}$, i.e. all $Z-Z-Z$ coupling coming with positive coefficients, this property will be lost. ${ }^{12}$ This shows that the form of $\mathcal{W}_{2}$ given in (A.12) is the correct one, and also fixes the relative signs between the terms in $\mathcal{W}_{4}$ involving $\Phi_{k}^{(1)}$ and $\Phi_{k}^{(2)}$.

We now turn to the second exchange symmetry, generated by the transformation $x^{6} \leftrightarrow$ $x^{8}, x^{7} \leftrightarrow x^{9}$ and $N_{2} \leftrightarrow N_{3}$. This exchanges the second and the third D-brane stacks. (A.15) shows that under this transformation $c^{(14)} \rightarrow-c^{(14)}, c^{(23)} \rightarrow c^{(23)}, c^{(12)} \leftrightarrow c^{(13)}$ and $c^{(24)} \leftrightarrow$ $c^{(34)}$. We can verify that all the $\mathcal{W}_{i}$ 's change sign if we accompany these transformations of $c^{(k \ell)}$ and $N_{i}$ with the following transformation on the fields:

$$
\begin{array}{rlrl}
\left(\Phi_{1}^{(4)}, \Phi_{1}^{(1)}\right) & \rightarrow\left(\Phi_{1}^{(4)}, \Phi_{1}^{(1)}\right), & \left(\Phi_{3}^{(1)}, \Phi_{3}^{(2)}\right) & \leftrightarrow\left(\Phi_{2}^{(1)}, \Phi_{2}^{(3)}\right), \\
\left(\Phi_{1}^{(2)}, \Phi_{1}^{(3)}\right) & \rightarrow\left(\Phi_{1}^{(3)}, \Phi_{1}^{(2)}\right), \quad\left(\Phi_{2}^{(4)}, \Phi_{2}^{(2)}\right) & \leftrightarrow-\left(\Phi_{3}^{(4)}, \Phi_{3}^{(3)}\right), \\
Z^{(41)} & \rightarrow Z^{(41)}, & Z^{(2 i)} & \leftrightarrow-Z^{(3 i)}, \\
Z^{(32)} & \leftrightarrow-Z^{(23)}, & Z^{(14)} & \rightarrow-Z^{(14)} .
\end{array}
$$

It follows from (A.17) and (A.18) that if for $i=1$ and $3, \Phi_{i}^{(i)}$ and $\Phi_{i}^{(4)}$ are interpreted as Wilson lines along the $w^{i}$ direction on the respective branes, then $\Phi_{2}^{(2)}$ and $\Phi_{2}^{(4)}$ should be interpreted as Wilson lines along the $-w^{2}$ direction on the D2-brane along the 6-7 directions and the D6-brane, respectively. This analysis also fixes the relative signs between the terms in $\mathcal{W}_{4}$ involving $\Phi_{k}^{(2)}$ and $\Phi_{k}^{(3)}$.

The theory under consideration also has a symmetry that exchanges brane stacks 1 and 4 , leaving the stacks 2 and 3 unchanged. This is induced by making a T-duality transformation along 6-7-8-9 directions and then performing an exchange $6 \leftrightarrow 8,7 \leftrightarrow 9$, and at the same time exchanging $N_{1}$ and $N_{4}$. We shall use the convention that, under a T-duality transformation along the $x^{m}$ direction, $g_{m n} \leftrightarrow b_{m n}$ to leading order in $g_{m n}$ and $b_{m n}$ for $n \neq m$. It is easy to see from (A.15) that under this exchange $c^{(14)}$ remains unchanged, $c^{(23)}$ changes sign, $c^{(12)} \leftrightarrow i c^{(24)}$ and $c^{(13)} \leftrightarrow i c^{(34)}$. It can be seen that the following accompanying transformation rules of the fields take $\mathcal{W}_{i}$ to $-\mathcal{W}_{i}$ for $1 \leq i \leq 4$ :

$$
\begin{aligned}
& \left(\Phi_{1}^{(4)}, \Phi_{1}^{(1)}\right) \rightarrow\left(\Phi_{1}^{(1)}, \Phi_{1}^{(4)}\right), \quad\left(\Phi_{2}^{(1)}, \Phi_{2}^{(3)}\right) \leftrightarrow i\left(\Phi_{3}^{(4)}, \Phi_{3}^{(3)}\right), \\
& \left(\Phi_{1}^{(2)}, \Phi_{1}^{(3)}\right) \rightarrow\left(\Phi_{1}^{(2)}, \Phi_{1}^{(3)}\right), \quad\left(\Phi_{2}^{(4)}, \Phi_{2}^{(2)}\right) \leftrightarrow i\left(\Phi_{3}^{(1)}, \Phi_{3}^{(2)}\right), \\
& Z^{(12)} \leftrightarrow-Z^{(42)}, \quad \quad Z^{(13)} \leftrightarrow-i Z^{(43)}, \quad Z^{(21)} \leftrightarrow-i Z^{(24)}, \quad Z^{(31)} \leftrightarrow-Z^{(34)}, \\
& Z^{(14)} \leftrightarrow i Z^{(41)}, \quad Z^{(32)} \rightarrow Z^{(32)}, \quad Z^{(23)} \rightarrow-Z^{(23)} .
\end{aligned}
$$

\footnotetext{
${ }^{12}$ Since the $c^{(k \ell)}$ 's are only determined up to phases in terms of the background fields, one may wonder whether the analysis that led to the conclusion that the choice of all positive signs in $\mathcal{W}_{2}$ is not allowed could be modified if we use $c^{(k \ell)}$ with different phases. To this end we note that the information that was needed to arrive at this result was that under the exchange $x^{4} \leftrightarrow x^{6}$ and $x^{5} \leftrightarrow x^{7}, c^{(12)}$ remains unchanged and $c^{(34)}$ changes sign. These transformation laws of $c^{(12)}$ and $c^{(34)}$ do not depend on the choice of phases of the $c^{(k \ell)}$ 's given in (A.15). Thus our argument holds.
} 
This fixes the relative signs between the terms in $\mathcal{W}_{4}$ involving $\Phi_{k}^{(1)}$ and $\Phi_{k}^{(4)}$. The factors of $i$ in the transformation laws relating $\Phi_{i}^{(k)}$ 's to $\Phi_{k}^{(k)}$ 's and $\Phi_{k}^{(4)}$ 's for $1 \leq i, k \leq 3, i \neq k$ show that if we interpret $\Phi_{i}^{(k)}$ as the position of the $k$-th D2-brane along $w^{i}$, then up to signs, $i \Phi_{i}^{(4)}$ and $i \Phi_{i}^{(i)}$ are to be interpreted as Wilson lines along $w^{i}$ on the $i$-th D2-brane and the D6-brane, respectively. Due to the comments below (A.18) it follows that there is a further factor of -1 in the definition of $\Phi_{2}^{(2)}$ and $\Phi_{2}^{(4)}$ relative to those for $\Phi_{i}^{(i)}$ and $\Phi_{i}^{(4)}$ for $i=1,3$.

All other exchange symmetries are compositions of the above three transformations and hence invariance of the potential under the former follows as a consequence of their invariance under the latter. Thus we see that the exchange symmetries together with judicious utilization of the field redefinition freedom fixes the signs of all the terms in the superpotential except for the overall sign of $\mathcal{W}_{4}$ relative to the other terms.

The superpotential determined this way also has other desired symmetries. For example we see from (A.15) that under the transformation $x^{4} \rightarrow x^{5}, x^{5} \rightarrow-x^{4}, c^{(12)}, c^{(13)}, c^{(24)}$ and $c^{(34)}$ get multiplied by $-i$ while the other $c^{(k \ell)}$ 's remain unchanged. It is easy to see that under this transformation the superpotential gets multiplied by an overall factor of $-i$, and hence leaves the potential invariant, if we transform the various fields as

$$
\begin{aligned}
\Phi_{1}^{(k)} & \rightarrow-i \Phi_{1}^{(k)} \quad \text { for } 1 \leq k \leq 4, & Z^{(12)} \rightarrow-i Z^{(12)}, \quad Z^{(13)} \rightarrow-i Z^{(13),} \\
Z^{(42)} & \rightarrow-i Z^{(42)}, & Z^{(43)} \rightarrow-i Z^{(43)},
\end{aligned}
$$

leaving the other fields invariant.

Finally consider the world-sheet parity transformation under which the NS-NS 2-form field changes sign. This by itself is not a symmetry of type IIA string theory, but becomes a symmetry if we accompany this by the parity transformation along the non-compact directions and $(-1)^{F_{L}}$ - this is simply the symmetry group by which we quotient the theory to generate orientifold 6-planes. Furthermore this transformation leaves the D6and D2-branes invariant. We see from (A.15) that under this transformation $c^{(k \ell)} \rightarrow c^{(k \ell)}$ and $c^{(k 4)} \rightarrow-c^{(k 4)}$ for $1 \leq k<\ell \leq 3$. It is easy to see that the following transformation on the fields combined with the above leaves the superpotential invariant:

$$
\begin{aligned}
& \Phi_{i}^{(4)} \rightarrow-\left(\Phi_{i}^{(4)}\right)^{T}, \quad \Phi_{i}^{(i)} \rightarrow-\left(\Phi_{i}^{(i)}\right)^{T}, \quad \Phi_{j}^{(i)} \rightarrow\left(\Phi_{j}^{(i)}\right)^{T} \quad \text { for } 1 \leq i, j \leq 3, j \neq i, \\
& Z^{(k \ell)} \rightarrow\left(Z^{(\ell k)}\right)^{T} \quad \text { for } 1 \leq k, \ell \leq 3, \ell \neq k, \\
& Z^{(14)} \rightarrow\left(Z^{(41)}\right)^{T}, \quad Z^{(24)} \rightarrow-\left(Z^{(42)}\right)^{T}, \quad Z^{(34)} \rightarrow\left(Z^{(43)}\right)^{T}, \\
& Z^{(41)} \rightarrow-\left(Z^{(14)}\right)^{T}, \quad Z^{(42)} \rightarrow\left(Z^{(24)}\right)^{T}, \quad Z^{(43)} \leftrightarrow-\left(Z^{(34)}\right)^{T} .
\end{aligned}
$$

The transposition operation involved in the transformation laws is a reflection of the fact that under world-sheet parity transformation open strings change their orientation.

Given the superpotential constructed above, the potential $V$ is given by (2.5)-(2.10). The shift symmetry (2.11) generalizes to

$$
\begin{aligned}
& \Phi_{m}^{(k)} \rightarrow \Phi_{m}^{(k)}+\xi_{m} I_{N_{k}}, \quad \text { for } \quad 1 \leq k \leq 3, \quad k \neq m ; \quad 1 \leq m \leq 3, \\
& \Phi_{k}^{(k)} \rightarrow \Phi_{k}^{(k)}+\zeta_{k} I_{N_{k}}, \quad \Phi_{k}^{(4)} \rightarrow \Phi_{k}^{(4)}+\zeta_{k} I_{N_{4}}, \quad \text { for } \quad 1 \leq k \leq 3, \\
& X_{i}^{(k)} \rightarrow X_{i}^{(k)}+a_{i} I_{N_{k}}, \quad \text { for } \quad 1 \leq i \leq 3 .
\end{aligned}
$$


We shall now argue that at a generic point in the moduli space, the vanishing of $V_{\text {gauge }}$ given in (2.9) requires all the $X_{i}^{(k)}$ 's to vanish (up to the shift symmetry described in the last line of (A.22)). To see this first note that the vanishing of $V_{\text {gauge }}$ requires each of the terms in (2.9) to vanish separately since each is a positive definite term. The vanishing of the last term tells us that $X_{i}^{(k)}$ and $X_{j}^{(k)}$ commute. Since $X_{i}^{(k)}$ 's are hermitian matrices, this implies that with the help of $\mathrm{U}\left(N_{k}\right)$ gauge transformations we can simultaneously diagonalize each $X_{i}^{(k)}$. Therefore we can take

$$
\left(X_{i}^{(k)}\right)_{m n}=a_{i, m}^{(k)} \delta_{m n}, \quad \text { for } \quad 1 \leq m, n \leq N_{k}, \quad 1 \leq k \leq 4
$$

Physically $a_{i, m}^{(k)}$ has the interpretation of the position along $x^{i}$ of the $m$-th brane in the $k$-th stack. Now requiring that the first two terms in (2.9) vanish we can easily see that

$$
\begin{aligned}
& \left(Z^{(k \ell)}\right)_{m n}\left(a_{i, m}^{(k)}-a_{i, n}^{(\ell)}\right)=0 \quad \text { for } 1 \leq m \leq N_{k}, 1 \leq n \leq N_{\ell}, 1 \leq i \leq 3, \\
& \left(\Phi^{(k)}\right)_{m n}\left(a_{i, m}^{(k)}-a_{i, n}^{(k)}\right)=0 \quad \text { for } 1 \leq m, n \leq N_{k}, 1 \leq i \leq 3 .
\end{aligned}
$$

This gives

$$
\begin{gathered}
\left(Z^{(k \ell)}\right)_{m n}=0 \quad \text { if } a_{i, m}^{(k)} \neq a_{i, n}^{(\ell)} \text { for any } i \text { for } 1 \leq m \leq N_{k}, 1 \leq n \leq N_{\ell}, \\
\left(\Phi^{(k)}\right)_{m n}=0 \quad \text { if } a_{i, m}^{(k)} \neq a_{i, n}^{(k)} \text { for any } i \text { for } 1 \leq m, n \leq N_{k} .
\end{gathered}
$$

Furthermore, once (A.25) is satisfied, there is no further constraint on the $a_{i, m}^{(k)}$ 's. Physically this means that if the $m$ 'th brane in the $k$-th stack and the $n$-th brane in the $\ell$-th stack are separated along the non-compact directions, then all fields associated with the open strings stretched between the two branes must have zero expectation value. Therefore we can divide the brane stacks into groups, where within each group all branes are coincident along the non-compact directions, and the brane stacks in two different groups are separated from each other along at least one of the non-compact directions. In this case all fields associated with open strings stretching between two different groups must vanish, and once this condition is satisfied we have no further constraint on the locations of the groups along the non-compact directions. In particular we can separate the different groups by arbitrary distance. If such a solution exists, then the mass of the total brane system will be given by the sum of the masses of the individual groups. But at a generic point in the moduli space away from subspaces of marginal stability this violates the BPS bound if the total charge vector is primitive, i.e. $\operatorname{gcd}\left\{N_{1}, N_{2}, N_{3}, N_{4}\right\}=1$. This shows that it should be impossible to satisfy the F- and D-term equations in this case. Therefore the only way to solve the equations is to set all the $a_{i, m}^{(k)}$ 's to be equal for any given $i$. Using the shift symmetry given in the last line of (A.22) we can set these to zero, showing that all the $X_{i}^{(k)}$ 's can be taken to vanish.

We can also see the absence of solutions with more than one group by directly analyzing the F-term equations. Let us consider for example the equations we get by setting the variation of $\mathcal{W}$ with respect to $\Phi_{3}^{(1)}$ and $\Phi_{3}^{(2)}$ to zero. Using (A.11)-(A.14) these equations take the form

$$
Z^{(12)} Z^{(21)}+N_{2} c^{(12)} I_{N_{1}}-\left[\Phi_{1}^{(1)}, \Phi_{2}^{(1)}\right]=0
$$




$$
Z^{(21)} Z^{(12)}+N_{1} c^{(12)} I_{N_{2}}+\left[\Phi_{1}^{(2)}, \Phi_{2}^{(2)}\right]=0
$$

Now suppose that we have a solution with multiple groups, with the first group containing $M_{1}$ D2-branes along 4-5 directions, $M_{2}$ D2-branes along 6-7 directions, $M_{3}$ D2-branes along 8-9 directions and $M_{4}$ D6-branes along 4-5-6-7-8-9 directions. Let us now take the trace of the first equation over the $M_{1}$ D2-branes in the 4-5 directions and the trace of the second equation over the $M_{2}$ D2-branes in the 6-7 directions. Since there are no components of $Z^{(k \ell)}$ or $\Phi_{m}^{(k)}$ with one leg in the first group and another leg in another group, the traces described above restrict the sum to be over the indices in the first group only. Denoting the trace with all indices in the first group by $\operatorname{Tr}_{1}$, we get

$$
\operatorname{Tr}_{1}\left(Z^{(12)} Z^{(21)}\right)+c^{(12)} N_{2} M_{1}=0, \quad \operatorname{Tr}_{1}\left(Z^{(21)} Z^{(12)}\right)+c^{(12)} N_{1} M_{2}=0 .
$$

Taking the difference between the two equations we see that we must have $N_{1} M_{2}=N_{2} M_{1}$. Repeating the analysis for other equations we get $N_{i} M_{j}=N_{j} M_{i}$ for $1 \leq i<j \leq 4$, or equivalently, $M_{i} / N_{i}=$ constant. The same analysis may be repeated for other groups, showing that the charge vector of each group must be proportional to the total charge vector. But this is impossible if the total charge vector is primitive. This shows that for primitive charge vector there are no solutions to the F-term equations with more than one group.

For completeness we also give the expected number of solutions to the F- and D-term constraints from the counting in the dual description [21]. For the $N_{i}$ 's satisfying the restriction

$$
\operatorname{gcd}\left\{N_{1} N_{2}, N_{1} N_{3}, N_{1} N_{4}, N_{2} N_{3}, N_{2} N_{4}, N_{3} N_{4}\right\}=1,
$$

this is given by [17]

$$
-\sum_{s \mid s_{0}} s \widehat{c}\left(4 N_{1} N_{2} N_{3} N_{4} / s^{2}\right)
$$

where $\widehat{c}(u)$ has been defined in $(2.13)$ and

$$
s_{0}=\prod_{\substack{i, j=1 \\ i<j}}^{4} \operatorname{gcd}\left\{N_{i}, N_{j}\right\} .
$$

Note that (A.30) is a slight rewriting of the corresponding expression given in [17].

\section{B Explicit solutions for $N=2$}

In this appendix we shall give the explicit solutions to the F- and D-term equations for the $N=2$ case. We give the results for the following choice of parameters:

$$
\begin{aligned}
& \left(c^{(12)}, c^{(13)}, c^{(14)}, c^{(23)}, c^{(24)}, c^{(34)}, c^{(1)}, c^{(2)}, c^{(3)}, c^{(4)}\right) \\
& =\left(\frac{2}{3}, \frac{3}{5}, \frac{5}{7}, \frac{7}{11}, \frac{11}{13}, \frac{13}{17}, \frac{17}{19}, \frac{19}{23}, \frac{23}{29},-\frac{31859}{25346}\right) .
\end{aligned}
$$

If we scale all the $c^{(k \ell)}$ 's and $c^{(k)}$ 's by a real positive parameter $\lambda$, then the solutions given below get scaled by an overall factor of $\sqrt{\lambda}$. As discussed in footnote 6 , we can justify the 
dropping of higher order terms in the superpotential and the periodicity of the variables $\Phi_{i}^{(k)}$ if we take the limit $\lambda \rightarrow 0$. This is the way we should interpret our solutions, although, in order to avoid cluttering, we shall refrain from displaying the factors of $\lambda$ and $\sqrt{\lambda}$ in the expressions for the parameters and solutions, respectively.

In the gauge (4.3) we give the 56 solutions below by specifying, for each solution, the variables in the following order:

$$
\begin{aligned}
& Z^{(12)}, Z^{(21)}, Z^{(13)}, Z^{(31)}, Z^{(23)}, Z^{(32)}, Z_{1}^{(14)}, Z_{2}^{(14)}, Z_{1}^{(41)}, Z_{2}^{(41)}, Z_{1}^{(24)}, Z_{2}^{(24)}, \\
& Z_{1}^{(42)}, Z_{2}^{(42)}, Z_{1}^{(34)}, Z_{2}^{(34)}, Z_{1}^{(43)}, Z_{2}^{(43)}, \Phi_{3}^{(2)}, \Phi_{1}^{(3)}, \Phi_{2}^{(3)}, \Phi_{1,11}^{(4)}, \Phi_{1,12}^{(4)}, \Phi_{1,21}^{(4)}, \Phi_{1,22}^{(4)}, \\
& \Phi_{2,11}^{(4)}, \Phi_{2,12}^{(4)}, \Phi_{2,21}^{(4)}, \Phi_{2,22}^{(4)}, \Phi_{3,11}^{(4)}, \Phi_{3,12}^{(4)}, \Phi_{3,21}^{(4)}, \Phi_{3,22}^{(4)} .
\end{aligned}
$$

The solutions can be organized by two $Z_{2}$ symmetries. The first $Z_{2}$ corresponds to complex conjugation of all the fields. Since the parameters $c^{(k \ell)}$ and $c^{(k)}$ have been chosen to be real, the F- and D-term constraints are invariant under complex conjugation and hence given a solution, its complex conjugate will also be a solution. The second $Z_{2}$ corresponds to a change in sign of all the fields under which the superpotential picks up an overall minus sign, and hence again the F- and D-term constraints are invariant under this transformation. However since the gauge conditions (4.5), (4.6) and (4.8) are not preserved by the second transformation, we have to accompany this with a compensating gauge transformation to restore the gauge. This can be done with the help of the element -1 of the second U(1) and the element $\operatorname{diag}(-1,1)$ of the $\mathrm{U}(2)$ group. It turns out that 12 of the 56 solutions are invariant under the product of the two $Z_{2}$ transformations - they are the first twelve among the solutions listed below. These solutions come in pairs related by the first $Z_{2}$ transformation. The others transform non-trivially under both $Z_{2}$ 's and hence come in groups of four, related by the $Z_{2} \times Z_{2}$ transformation. The full set of solutions are:

$1.1031,-0.60435,0.48800,-1.2295,1.0504,-0.60582,1.3763,-0.63328,-0.73151,0.66599,0,1.4940$, $0.086853,-1.1327,0.99557,-0.83985,-1.0579,0.56703,0.49066,0.52570,1.3450,-0.66999,1.1383$, $-0.68895,-0.77563,0.46310,0.18210,0.14322,-0.33432,-0.75607,-0.62011,1.1187,-0.58452$,

$1.1031,-0.60435,-0.48800,1.2295,1.0504,-0.60582,1.3763,0.63328,-0.73151,-0.66599,0,1.4940$, $-0.086853,-1.1327,0.99557,0.83985,-1.0579,-0.56703,-0.49066,-0.52570,-1.3450,0.66999$, $1.1383,-0.68895,0.77563,-0.46310,0.18210,0.14322,0.33432,0.75607,-0.62011,1.1187,0.58452$,

$0.61097,-1.0912,1.2046 i, 0.49808 i, 0.62251,-1.0223,1.4463,0.72947 i,-0.26673,1.4295 i, 0,1.4840$, $0.48529 i,-1.1404,1.0588,-1.3941 i,-1.3097,-0.10235 i,-2.2283 i,-2.3555 i,-1.1947 i,-0.85898 i$, $-0.98702,-0.045414,-0.89763 i,-2.9804 i, 0.23714,-0.61928,-1.1212 i,-0.46046 i,-0.19871$, $-0.86642,-1.1979 i$,

$0.61097,-1.0912,-1.2046 i,-0.49808 i, 0.62251,-1.0223,1.4463,-0.72947 i,-0.26673,-1.4295 i, 0$, $1.4840,-0.48529 i,-1.1404,1.0588,1.3941 i,-1.3097,0.10235 i, 2.2283 i, 2.3555 i, 1.1947 i, 0.85898 i$, $-0.98702,-0.045414,0.89763 i, 2.9804 i, 0.23714,-0.61928,1.1212 i, 0.46046 i,-0.19871,-0.86642$, $1.1979 i$

$0.73263,-0.90996,1.1484 i, 0.52249 i, 0.73592,-0.86472,1.0930,0.72576 i,-0.55912,1.1263 i, 0$, $1.4575,0.18706 i,-1.1611,1.0416,-1.2563 i,-0.89879,-0.47215 i,-2.2265 i,-2.3550 i,-0.087600 i$, $-0.82484 i,-0.97754,-0.40590,-0.95563 i,-2.2487 i, 0.045714,-0.15648,-1.0875 i,-0.67719 i$, $-0.36232,-1.0160,-1.0046 i$ 
$0.73263,-0.90996,-1.1484 i,-0.52249 i, 0.73592,-0.86472,1.0930,-0.72576 i,-0.55912,-1.1263 i, 0$, $1.4575,-0.18706 i,-1.1611,1.0416,1.2563 i,-0.89879,0.47215 i, 2.2265 i, 2.3550 i, 0.087600 i, 0.82484 i$, $-0.97754,-0.40590,0.95563 i, 2.2487 i, 0.045714,-0.15648,1.0875 i, 0.67719 i,-0.36232,-1.0160$, $1.0046 i$

$0.66655,-1.0002,0.75311 i, 0.79669 i, 0.59925,-1.0619,1.0227,-1.3300 i, 0.080251,-1.1358 i, 0$, $1.5175,0.14385 i,-1.1152,-1.1436,-0.73340 i, 1.3732,0.055941 i, 1.2460 i,-1.1153 i, 2.4939 i, 0.92246 i$, $-0.77136,0.061744,0.93839 i, 1.9135 i, 1.0129,-1.1256,0.58697 i, 0.0064870 i, 0.30129,-1.1211$, $-0.28274 i$,

$0.66655,-1.0002,-0.75311 i,-0.79669 i, 0.59925,-1.0619,1.0227,1.3300 i, 0.080251,1.1358 i, 0$, $1.5175,-0.14385 i,-1.1152,-1.1436,0.73340 i, 1.3732,-0.055941 i,-1.2460 i, 1.1153 i,-2.4939 i$, $-0.92246 i,-0.77136,0.061744,-0.93839 i,-1.9135 i, 1.0129,-1.1256,-0.58697 i,-0.0064870 i$, $0.30129,-1.1211,0.28274 i$,

$0.68357,-0.97528,-0.78353 i,-0.76577 i, 0.69051,-0.92159,2.0781,-0.27085 i,-0.88339,1.5034 i, 0$, $1.7460,-1.1808 i,-0.96926,-0.70695,-1.8244 i,-1.2693,-1.3301 i,-2.1494 i,-2.2566 i, 3.2237 i$, $-0.29046 i, 1.2418,-0.18345,0.15652 i, 1.0460 i, 0.69047,-1.4404,-0.57024 i, 0.67798 i, 0.48642$, $0.60953,-0.80716 i$,

$0.68357,-0.97528,0.78353 i, 0.76577 i, 0.69051,-0.92159,2.0781,0.27085 i,-0.88339,-1.5034 i, 0$, $1.7460,1.1808 i,-0.96926,-0.70695,1.8244 i,-1.2693,1.3301 i, 2.1494 i, 2.2566 i,-3.2237 i, 0.29046 i$, $1.2418,-0.18345,-0.15652 i,-1.0460 i, 0.69047,-1.4404,0.57024 i,-0.67798 i, 0.48642,0.60953$, $0.80716 i$,

$0.59598,-1.1186,-0.73969 i,-0.81115 i, 0.65823,-0.96678,1.6985,0.30140 i,-0.92427,-0.46869 i, 0$, $1.3919,0.16684 i,-1.2158,0.29842,-1.4089 i, 0.63575,-1.2202 i, 1.0605 i,-1.3233 i,-2.4873 i, 0.19243 i$, $1.1190,-0.35198,0.095824 i,-1.6071 i, 1.1791,-1.2238,-0.90847 i,-0.98383 i,-0.042304,0.76948$, $-0.77265 i$,

$0.59598,-1.1186,0.73969 i, 0.81115 i, 0.65823,-0.96678,1.6985,-0.30140 i,-0.92427,0.46869 i, 0$, $1.3919,-0.16684 i,-1.2158,0.29842,1.4089 i, 0.63575,1.2202 i,-1.0605 i, 1.3233 i, 2.4873 i,-0.19243 i$, $1.1190,-0.35198,-0.095824 i, 1.6071 i, 1.1791,-1.2238,0.90847 i, 0.98383 i,-0.042304,0.76948$, $0.77265 i$,

$0.45313, \quad-1.4712, \quad 0.56822-0.90538 i, \quad-0.29838-0.47544 i, \quad 0.44418, \quad-1.4327, \quad 1.6133$, $-0.28628+0.85922 i, \quad-1.06265-0.20046 i, \quad 0.23903-0.41227 i, \quad 0, \quad 1.4603, \quad 0.25762-0.03156 i$, $-1.1589, \quad-1.18478-0.54983 i, \quad-0.58503+0.44603 i, \quad 1.29014-0.03809 i, \quad 0.52573-0.73456 i$, $-0.14716+0.55274 i, \quad-0.15369+0.58393 i, \quad-0.8983-2.4883 i, \quad 0.64477+0.07407 i$, $0.51341-0.56880 i,-0.96725+0.17004 i, 0.22399+0.20236 i,-0.12969-0.36193 i, 1.9718-0.0456 i$, $-1.9858-0.1672 i, 0.11047-0.72999 i, 0.50307+0.02573 i, 0.94973-0.33977 i,-0.48769+0.41436 i$, $0.30881+0.23653 i$

$0.45313, \quad-1.4712, \quad 0.56822+0.90538 i, \quad-0.29838+0.47544 i, \quad 0.44418, \quad-1.4327, \quad 1.6133$, $-0.28628-0.85922 i, \quad-1.06265+0.20046 i, \quad 0.23903+0.41227 i, \quad 0, \quad 1.4603, \quad 0.25762+0.03156 i$, $-1.1589, \quad-1.18478+0.54983 i, \quad-0.58503-0.44603 i, \quad 1.29014+0.03809 i, \quad 0.52573+0.73456 i$, $-0.14716-0.55274 i, \quad-0.15369-0.58393 i, \quad-0.8983+2.4883 i, \quad 0.64477-0.07407 i$, $0.51341+0.56880 i,-0.96725-0.17004 i, 0.22399-0.20236 i,-0.12969+0.36193 i, 1.9718+0.0456 i$, $-1.9858+0.1672 i, 0.11047+0.72999 i, 0.50307-0.02573 i, 0.94973+0.33977 i,-0.48769-0.41436 i$, $0.30881-0.23653 i$ 
$0.45313, \quad-1.4712, \quad-0.56822+0.90538 i, \quad 0.29838+0.47544 i, \quad 0.44418, \quad-1.4327, \quad 1.6133$, $0.28628-0.85922 i, \quad-1.06265-0.20046 i, \quad-0.23903+0.41227 i, \quad 0, \quad 1.4603, \quad-0.25762+0.03156 i$, $-1.1589, \quad-1.18478-0.54983 i, \quad 0.58503-0.44603 i, \quad 1.29014-0.03809 i, \quad-0.52573+0.73456 i$, $0.14716-0.55274 i, 0.15369-0.58393 i, 0.8983+2.4883 i,-0.64477-0.07407 i, 0.51341-0.56880 i$, $-0.96725+0.17004 i,-0.22399-0.20236 i, 0.12969+0.36193 i, 1.9718-0.0456 i,-1.9858-0.1672 i$, $-0.11047+0.72999 i, \quad-0.50307-0.02573 i, \quad 0.94973-0.33977 i, \quad-0.48769+0.41436 i$, $-0.30881-0.23653 i$,

$0.45313, \quad-1.4712, \quad-0.56822-0.90538 i, \quad 0.29838-0.47544 i, \quad 0.44418, \quad-1.4327, \quad 1.6133$, $0.28628+0.85922 i, \quad-1.06265+0.20046 i, \quad-0.23903-0.41227 i, \quad 0, \quad 1.4603, \quad-0.25762-0.03156 i$, $-1.1589, \quad-1.18478+0.54983 i, \quad 0.58503+0.44603 i, \quad 1.29014+0.03809 i, \quad-0.52573-0.73456 i$, $0.14716+0.55274 i, \quad 0.15369+0.58393 i, \quad 0.8983-2.4883 i,-0.64477+0.07407 i, \quad 0.51341+0.56880 i$, $-0.96725-0.17004 i,-0.22399+0.20236 i, 0.12969-0.36193 i, 1.9718+0.0456 i,-1.9858+0.1672 i$, $-0.11047-0.72999 i, \quad-0.50307+0.02573 i, \quad 0.94973+0.33977 i, \quad-0.48769-0.41436 i$, $-0.30881+0.23653 i$

$0.95002, \quad-0.70174, \quad-0.32710+0.46207 i, \quad 0.61236+0.86502 i, \quad 0.91139, \quad-0.69823, \quad 1.4042$, $0.69607+0.01764 i, \quad-1.07310+0.03164 i, \quad 0.110778-0.066634 i, \quad 0, \quad 1.5414, \quad-0.32109-0.41749 i$, $-1.0979, \quad 0.55005+1.21024 i, \quad 0.16063-0.43612 i, \quad-0.57733+0.76664 i, \quad-0.77052-0.36748 i$, $-1.0386-0.9771 i, \quad-1.0513-0.9773 i, \quad-1.6481+0.1394 i, \quad 0.75969-0.23579 i, \quad 0.16464-0.02762 i$, $-0.45318+0.69073 i, 1.5501-0.2952 i, 0.21242-0.81845 i, 0.18804+0.61876 i,-0.31405+0.71559 i$, $-0.22192-0.26590 i, \quad 1.13165-0.17765 i, \quad 0.54413+0.83642 i, \quad 0.132118+0.002732 i$, $1.05645-0.27973 i$,

$0.95002, \quad-0.70174, \quad-0.32710-0.46207 i, \quad 0.61236-0.86502 i, \quad 0.91139, \quad-0.69823, \quad 1.4042$, $0.69607-0.01764 i, \quad-1.07310-0.03164 i, \quad 0.110778+0.066634 i, \quad 0, \quad 1.5414,-0.32109+0.41749 i$, $-1.0979, \quad 0.55005-1.21024 i, \quad 0.16063+0.43612 i, \quad-0.57733-0.76664 i, \quad-0.77052+0.36748 i$, $-1.0386+0.9771 i, \quad-1.0513+0.9773 i, \quad-1.6481-0.1394 i, \quad 0.75969+0.23579 i, \quad 0.16464+0.02762 i$, $-0.45318-0.69073 i, 1.5501+0.2952 i, 0.21242+0.81845 i, 0.18804-0.61876 i,-0.31405-0.71559 i$, $-0.22192+0.26590 i, \quad 1.13165+0.17765 i, \quad 0.54413-0.83642 i, \quad 0.132118-0.002732 i$, $1.05645+0.27973 i$

$0.95002, \quad-0.70174, \quad 0.32710-0.46207 i, \quad-0.61236-0.86502 i, \quad 0.91139, \quad-0.69823, \quad 1.4042$, $-0.69607-0.01764 i,-1.07310+0.03164 i,-0.110778+0.066634 i, \quad 0, \quad 1.5414, \quad 0.32109+0.41749 i$, $-1.0979, \quad 0.55005+1.21024 i, \quad-0.16063+0.43612 i, \quad-0.57733+0.76664 i, \quad 0.77052+0.36748 i$, $1.0386+0.9771 i, \quad 1.0513+0.9773 i, \quad 1.6481-0.1394 i, \quad-0.75969+0.23579 i, \quad 0.16464-0.02762 i$, $-0.45318+0.69073 i, \quad-1.5501+0.2952 i, \quad-0.21242+0.81845 i, \quad 0.18804+0.61876 i$, $-0.31405+0.71559 i, \quad 0.22192+0.26590 i, \quad-1.13165+0.17765 i, \quad 0.54413+0.83642 i$, $0.132118+0.002732 i,-1.05645+0.27973 i$

$0.95002, \quad-0.70174, \quad 0.32710+0.46207 i, \quad-0.61236+0.86502 i, \quad 0.91139, \quad-0.69823, \quad 1.4042$, $-0.69607+0.01764 i,-1.07310-0.03164 i,-0.110778-0.066634 i, \quad 0, \quad 1.5414,0.32109-0.41749 i$, $-1.0979, \quad 0.55005-1.21024 i, \quad-0.16063-0.43612 i, \quad-0.57733-0.76664 i, \quad 0.77052-0.36748 i$, $1.0386-0.9771 i, \quad 1.0513-0.9773 i, \quad 1.6481+0.1394 i, \quad-0.75969-0.23579 i, \quad 0.16464+0.02762 i$, $-0.45318-0.69073 i, \quad-1.5501-0.2952 i, \quad-0.21242-0.81845 i, \quad 0.18804-0.61876 i$, $-0.31405-0.71559 i, \quad 0.22192-0.26590 i, \quad-1.13165-0.17765 i$, $0.54413-0.83642 i$

$0.132118-0.002732 i,-1.05645-0.27973 i$,

$0.91406, \quad-0.72935, \quad-0.32785+0.21228 i, \quad 1.2895+0.8349 i, \quad 1.4439, \quad-0.44072, \quad 1.9777$, $0.59874-0.39052 i, \quad-0.71679+0.35810 i, \quad 0.52839-0.83821 i, \quad 0, \quad 1.5872, \quad-1.4616-0.0841 i$, $-1.0662, \quad 0.53349+0.85291 i, \quad 0.25457-0.94589 i, \quad-0.54140+0.29559 i, \quad-0.56202-0.89376 i$, $-3.7027+0.1711 i, \quad-0.45217-1.08965 i, \quad-1.31223-0.02937 i, \quad 0.28581-0.03529 i$, $0.28570+0.19367 i,-0.42571+0.11678 i, 1.4713-0.2882 i, 0.25631-0.43236 i, 0.00524+0.38763 i$, $-0.42348+0.77593 i,-0.04355-0.68106 i, 3.3594-0.7888 i, 1.36463+0.09310 i, 0.80459-0.12635 i$, $1.13364-0.56938 i$ 
$0.91406, \quad-0.72935, \quad-0.32785-0.21228 i, \quad 1.2895-0.8349 i, \quad 1.4439, \quad-0.44072, \quad 1.9777$, $0.59874+0.39052 i, \quad-0.71679-0.35810 i, \quad 0.52839+0.83821 i, \quad 0, \quad 1.5872, \quad-1.4616+0.0841 i$, $-1.0662, \quad 0.53349-0.85291 i, \quad 0.25457+0.94589 i, \quad-0.54140-0.29559 i, \quad-0.56202+0.89376 i$, $-3.7027-0.1711 i, \quad-0.45217+1.08965 i, \quad-1.31223+0.02937 i, \quad 0.28581+0.03529 i$, $0.28570-0.19367 i,-0.42571-0.11678 i, \quad 1.4713+0.2882 i, \quad 0.25631+0.43236 i, 0.00524-0.38763 i$, $-0.42348-0.77593 i,-0.04355+0.68106 i, 3.3594+0.7888 i, 1.36463-0.09310 i, 0.80459+0.12635 i$, $1.13364+0.56938 i$

$0.91406, \quad-0.72935, \quad 0.32785-0.21228 i, \quad-1.2895-0.8349 i, \quad 1.4439, \quad-0.44072, \quad 1.9777$, $-0.59874+0.39052 i, \quad-0.71679+0.35810 i, \quad-0.52839+0.83821 i, \quad 0, \quad 1.5872, \quad 1.4616+0.0841 i$, $-1.0662, \quad 0.53349+0.85291 i, \quad-0.25457+0.94589 i, \quad-0.54140+0.29559 i, \quad 0.56202+0.89376 i$, $3.7027-0.1711 i, \quad 0.45217+1.08965 i, 1.31223+0.02937 i,-0.28581+0.03529 i, 0.28570+0.19367 i$, $-0.42571+0.11678 i, \quad-1.4713+0.2882 i, \quad-0.25631+0.43236 i, \quad 0.00524+0.38763 i$, $-0.42348+0.77593 i, 0.04355+0.68106 i,-3.3594+0.7888 i, 1.36463+0.09310 i, 0.80459-0.12635 i$, $-1.13364+0.56938 i$,

$0.91406, \quad-0.72935, \quad 0.32785+0.21228 i, \quad-1.2895+0.8349 i, \quad 1.4439, \quad-0.44072, \quad 1.9777$, $-0.59874-0.39052 i, \quad-0.71679-0.35810 i, \quad-0.52839-0.83821 i, \quad 0, \quad 1.5872, \quad 1.4616-0.0841 i$, $-1.0662, \quad 0.53349-0.85291 i, \quad-0.25457-0.94589 i, \quad-0.54140-0.29559 i, \quad 0.56202-0.89376 i$, $3.7027+0.1711 i, 0.45217-1.08965 i, 1.31223-0.02937 i,-0.28581-0.03529 i, 0.28570-0.19367 i$, $-0.42571-0.11678 i, \quad-1.4713-0.2882 i, \quad-0.25631-0.43236 i, \quad 0.00524-0.38763 i$, $-0.42348-0.77593 i, 0.04355-0.68106 i,-3.3594-0.7888 i, 1.36463-0.09310 i, 0.80459+0.12635 i$, $-1.13364-0.56938 i$,

$1.6191, \quad-0.41174, \quad-0.31539+0.21907 i, \quad 1.2832+0.8914 i, \quad 0.89136, \quad-0.71393, \quad 1.1087$, $0.83973+0.29799 i, \quad-1.00374+0.24552 i, \quad-0.43614-0.16939 i, \quad 0, \quad 2.2206, \quad-0.24786-1.13810 i$, $-0.76211, \quad 0.9500+1.2734 i, \quad 0.22293+0.17133 i, \quad-0.27088+0.66960 i, \quad-1.8137+0.0879 i$, $-0.42589-1.01614 i, \quad-4.3726+0.1178 i, \quad-1.27855-0.01388 i, \quad 0.94073-0.59690 i$, $-0.10924-0.54527 i,-0.17013+1.07890 i, 4.2738-0.7905 i, 0.27296-1.10597 i, 0.13837+0.05770 i$, $0.17575+0.51118 i, \quad-0.066218+0.013520 i, \quad 1.05447-0.31776 i, \quad 0.18575+0.84116 i$, $-0.06819-0.18417 i, 0.54875+0.00570 i$

$1.6191, \quad-0.41174, \quad-0.31539-0.21907 i, \quad 1.2832-0.8914 i, \quad 0.89136, \quad-0.71393, \quad 1.1087$, $0.83973-0.29799 i, \quad-1.00374-0.24552 i, \quad-0.43614+0.16939 i, \quad 0, \quad 2.2206, \quad-0.24786+1.13810 i$, $-0.76211, \quad 0.9500-1.2734 i, \quad 0.22293-0.17133 i, \quad-0.27088-0.66960 i, \quad-1.8137-0.0879 i$, $-0.42589+1.01614 i, \quad-4.3726-0.1178 i, \quad-1.27855+0.01388 i, \quad 0.94073+0.59690 i$, $-0.10924+0.54527 i,-0.17013-1.07890 i, 4.2738+0.7905 i, 0.27296+1.10597 i, 0.13837-0.05770 i$, $0.17575-0.51118 i, \quad-0.066218-0.013520 i, \quad 1.05447+0.31776 i, \quad 0.18575-0.84116 i$, $-0.06819+0.18417 i, 0.54875-0.00570 i$,

$1.6191, \quad-0.41174, \quad 0.31539-0.21907 i, \quad-1.2832-0.8914 i, \quad 0.89136, \quad-0.71393, \quad 1.1087$, $-0.83973-0.29799 i, \quad-1.00374+0.24552 i, \quad 0.43614+0.16939 i, \quad 0, \quad 2.2206, \quad 0.24786+1.13810 i$, $-0.76211, \quad 0.9500+1.2734 i, \quad-0.22293-0.17133 i, \quad-0.27088+0.66960 i, \quad 1.8137-0.0879 i$, $0.42589+1.01614 i, 4.3726-0.1178 i, 1.27855+0.01388 i,-0.94073+0.59690 i,-0.10924-0.54527 i$, $-0.17013+1.07890 i, \quad-4.2738+0.7905 i, \quad-0.27296+1.10597 i, \quad 0.13837+0.05770 i$, $0.17575+0.51118 i, \quad 0.066218-0.013520 i, \quad-1.05447+0.31776 i, \quad 0.18575+0.84116 i$, $-0.06819-0.18417 i,-0.54875-0.00570 i$,

$1.6191, \quad-0.41174, \quad 0.31539+0.21907 i, \quad-1.2832+0.8914 i, \quad 0.89136, \quad-0.71393, \quad 1.1087$, $-0.83973+0.29799 i, \quad-1.00374-0.24552 i, \quad 0.43614-0.16939 i, \quad 0, \quad 2.2206, \quad 0.24786-1.13810 i$, $-0.76211, \quad 0.9500-1.2734 i, \quad-0.22293+0.17133 i, \quad-0.27088-0.66960 i, \quad 1.8137+0.0879 i$, $0.42589-1.01614 i, 4.3726+0.1178 i, 1.27855-0.01388 i,-0.94073-0.59690 i,-0.10924+0.54527 i$, $-0.17013-1.07890 i, \quad-4.2738-0.7905 i, \quad-0.27296-1.10597 i, \quad 0.13837-0.05770 i$, $0.17575-0.51118 i, \quad 0.066218+0.013520 i, \quad-1.05447-0.31776 i, \quad 0.18575-0.84116 i$, $-0.06819+0.18417 i,-0.54875+0.00570 i$ 
$1.9767, \quad-0.33727, \quad 0.05613-0.20931 i, \quad-0.7171-2.6743 i, \quad 1.8266, \quad-0.34838, \quad 2.5896$, $-0.97646+0.06703 i,-0.50833+0.57791 i, 0.0097+1.5333 i, 0,3.0381,2.6956+0.4993 i,-0.55702$, $0.2200+1.8898 i, \quad-0.39511+0.29393 i, \quad-0.34960+0.14642 i, \quad 2.6774+0.4012 i, \quad 3.7967+0.6721 i$, $4.4427+0.7059 i, 0.66723-0.48517 i,-0.31466+0.03542 i, 0.56011+0.26856 i,-0.42542+0.12618 i$, $-4.6583+0.4940 i, \quad-0.25449+0.46452 i, \quad-0.062714+0.060676 i, \quad-0.15517+1.13620 i$, $-0.12916+0.16928 i,-3.7951+0.4902 i, 1.3886+0.3523 i, 0.32390-0.11002 i,-0.46294+0.00590 i$,

$1.9767, \quad-0.33727, \quad 0.05613+0.20931 i, \quad-0.7171+2.6743 i, \quad 1.8266, \quad-0.34838, \quad 2.5896$, $-0.97646-0.06703 i,-0.50833-0.57791 i, 0.0097-1.5333 i, 0,3.0381,2.6956-0.4993 i,-0.55702$, $0.2200-1.8898 i, \quad-0.39511-0.29393 i, \quad-0.34960-0.14642 i, \quad 2.6774-0.4012 i, \quad 3.7967-0.6721 i$, $4.4427-0.7059 i, 0.66723+0.48517 i,-0.31466-0.03542 i, 0.56011-0.26856 i,-0.42542-0.12618 i$, $-4.6583-0.4940 i, \quad-0.25449-0.46452 i, \quad-0.062714-0.060676 i, \quad-0.15517-1.13620 i$, $-0.12916-0.16928 i,-3.7951-0.4902 i, 1.3886-0.3523 i, 0.32390+0.11002 i,-0.46294-0.00590 i$,

$1.9767, \quad-0.33727, \quad-0.05613+0.20931 i, \quad 0.7171+2.6743 i, \quad 1.8266, \quad-0.34838, \quad 2.5896$, $0.97646-0.06703 i, \quad-0.50833+0.57791 i, \quad-0.0097-1.5333 i, \quad 0, \quad 3.0381, \quad-2.6956-0.4993 i$, $-0.55702, \quad 0.2200+1.8898 i, \quad 0.39511-0.29393 i, \quad-0.34960+0.14642 i, \quad-2.6774-0.4012 i$, $-3.7967-0.6721 i,-4.4427-0.7059 i,-0.66723+0.48517 i, 0.31466-0.03542 i, 0.56011+0.26856 i$, $-0.42542+0.12618 i, \quad 4.6583-0.4940 i, \quad 0.25449-0.46452 i, \quad-0.062714+0.060676 i$, $-0.15517+1.13620 i, \quad 0.12916-0.16928 i, \quad 3.7951-0.4902 i, \quad 1.3886+0.3523 i, \quad 0.32390-0.11002 i$, $0.46294-0.00590 i$

$1.9767, \quad-0.33727, \quad-0.05613-0.20931 i, \quad 0.7171-2.6743 i, \quad 1.8266, \quad-0.34838, \quad 2.5896$, $0.97646+0.06703 i, \quad-0.50833-0.57791 i, \quad-0.0097+1.5333 i, \quad 0, \quad 3.0381, \quad-2.6956+0.4993 i$, $-0.55702, \quad 0.2200-1.8898 i, \quad 0.39511+0.29393 i, \quad-0.34960-0.14642 i, \quad-2.6774+0.4012 i$, $-3.7967+0.6721 i,-4.4427+0.7059 i,-0.66723-0.48517 i, 0.31466+0.03542 i, 0.56011-0.26856 i$, $-0.42542-0.12618 i, \quad 4.6583+0.4940 i, \quad 0.25449+0.46452 i, \quad-0.062714-0.060676 i$, $-0.15517-1.13620 i, \quad 0.12916+0.16928 i, \quad 3.7951+0.4902 i, \quad 1.3886-0.3523 i, \quad 0.32390+0.11002 i$, $0.46294+0.00590 i$

$1.9110, \quad-0.34887, \quad 0.00327-0.22368 i, \quad-0.0393-2.6819 i, \quad 1.7784, \quad-0.35783, \quad 2.6804$, $-0.53916+0.27523 i, \quad-0.27538+0.28432 i, \quad 0.4435+1.6399 i, \quad 0, \quad 3.0545, \quad-2.7364-0.4622 i$, $-0.55405, \quad 0.2485-1.8820 i, \quad-0.15028-0.62950 i, \quad-0.84974-0.35066 i, \quad 2.5088-0.4469 i$, $-3.6825-0.6863 i, \quad 4.3083-0.7236 i,-0.03074+0.48708 i, \quad 0.24037-0.02620 i, 1.36785+0.30507 i$, $0.42890-0.02582 i, \quad-4.0393-0.4868 i, \quad 0.03734-0.23398 i, \quad 0.021371-0.082685 i$, $-0.16148-1.09578 i, \quad-0.02592-0.39795 i, \quad 3.6366-0.4677 i, \quad-0.64660-0.11788 i$, $0.38877-0.11146 i,-0.38953-0.01540 i$,

$1.9110, \quad-0.34887, \quad 0.00327+0.22368 i, \quad-0.0393+2.6819 i, \quad 1.7784, \quad-0.35783, \quad 2.6804$, $-0.53916-0.27523 i, \quad-0.27538-0.28432 i, \quad 0.4435-1.6399 i, \quad 0, \quad 3.0545, \quad-2.7364+0.4622 i$, $-0.55405, \quad 0.2485+1.8820 i, \quad-0.15028+0.62950 i, \quad-0.84974+0.35066 i, \quad 2.5088+0.4469 i$, $-3.6825+0.6863 i, \quad 4.3083+0.7236 i, \quad-0.03074-0.48708 i, \quad 0.24037+0.02620 i, \quad 1.36785-0.30507 i$, $0.42890+0.02582 i, \quad-4.0393+0.4868 i, \quad 0.03734+0.23398 i, \quad 0.021371+0.082685 i$, $-0.16148+1.09578 i, \quad-0.02592+0.39795 i, \quad 3.6366+0.4677 i, \quad-0.64660+0.11788 i$, $0.38877+0.11146 i,-0.38953+0.01540 i$

(B.36)

$1.9110, \quad-0.34887, \quad-0.00327+0.22368 i, \quad 0.0393+2.6819 i, \quad 1.7784, \quad-0.35783, \quad 2.6804$, $0.53916-0.27523 i,-0.27538+0.28432 i,-0.4435-1.6399 i, 0,3.0545,2.7364+0.4622 i,-0.55405$, $0.2485-1.8820 i, \quad 0.15028+0.62950 i, \quad-0.84974-0.35066 i, \quad-2.5088+0.4469 i, \quad 3.6825+0.6863 i$, $-4.3083+0.7236 i, 0.03074-0.48708 i,-0.24037+0.02620 i, 1.36785+0.30507 i, 0.42890-0.02582 i$, $4.0393+0.4868 i, \quad-0.03734+0.23398 i, \quad 0.021371-0.082685 i, \quad-0.16148-1.09578 i$, $0.02592+0.39795 i,-3.6366+0.4677 i,-0.64660-0.11788 i, 0.38877-0.11146 i, 0.38953+0.01540 i$, 
$1.9110, \quad-0.34887, \quad-0.00327-0.22368 i, \quad 0.0393-2.6819 i, \quad 1.7784, \quad-0.35783, \quad 2.6804$, $0.53916+0.27523 i,-0.27538-0.28432 i,-0.4435+1.6399 i, 0,3.0545,2.7364-0.4622 i,-0.55405$, $0.2485+1.8820 i, \quad 0.15028-0.62950 i, \quad-0.84974+0.35066 i, \quad-2.5088-0.4469 i, \quad 3.6825-0.6863 i$, $-4.3083-0.7236 i, 0.03074+0.48708 i,-0.24037-0.02620 i, 1.36785-0.30507 i, 0.42890+0.02582 i$, $4.0393-0.4868 i, \quad-0.03734-0.23398 i, \quad 0.021371+0.082685 i, \quad-0.16148+1.09578 i$, $0.02592-0.39795 i,-3.6366-0.4677 i,-0.64660+0.11788 i, 0.38877+0.11146 i, 0.38953-0.01540 i$,

$1.4754, \quad-0.45185, \quad 0.26993-0.31443 i, \quad-0.9431-1.0986 i, \quad 0.90647, \quad-0.70202, \quad 1.1900$, $-0.64403+0.44663 i, \quad-0.70679+0.19227 i, \quad 0.44962+0.66707 i, \quad 0, \quad 2.1641, \quad-0.58808-1.12062 i$, $-0.78201, \quad-0.5172-1.5098 i, \quad-0.13148-0.42354 i, \quad-0.15008-0.87223 i, \quad 1.6534-0.1717 i$, $-0.26139-0.99093 i, \quad 3.8126-0.2532 i, \quad-0.61422-0.01569 i, \quad 0.40219-0.56716 i$, $0.78585+1.09294 i, 0.52651-1.06313 i,-3.4366-0.4772 i,-0.20918-0.81249 i, 0.19179-0.23502 i$, $-0.46509-0.63244 i, 0.07940-0.27067 i, 0.96433-0.28726 i, 0.02610-0.29483 i, 0.12662-0.40894 i$, $-0.39814-0.03508 i$,

$1.4754, \quad-0.45185, \quad 0.26993+0.31443 i, \quad-0.9431+1.0986 i, \quad 0.90647, \quad-0.70202, \quad 1.1900$, $-0.64403-0.44663 i, \quad-0.70679-0.19227 i, \quad 0.44962-0.66707 i, \quad 0, \quad 2.1641, \quad-0.58808+1.12062 i$, $-0.78201, \quad-0.5172+1.5098 i, \quad-0.13148+0.42354 i, \quad-0.15008+0.87223 i, \quad 1.6534+0.1717 i$, $-0.26139+0.99093 i, \quad 3.8126+0.2532 i, \quad-0.61422+0.01569 i, \quad 0.40219+0.56716 i$, $0.78585-1.09294 i, 0.52651+1.06313 i,-3.4366+0.4772 i,-0.20918+0.81249 i, 0.19179+0.23502 i$, $-0.46509+0.63244 i, 0.07940+0.27067 i, 0.96433+0.28726 i, 0.02610+0.29483 i, 0.12662+0.40894 i$, $-0.39814+0.03508 i$,

$1.4754, \quad-0.45185, \quad-0.26993+0.31443 i, \quad 0.9431+1.0986 i, \quad 0.90647, \quad-0.70202, \quad 1.1900$, $0.64403-0.44663 i, \quad-0.70679+0.19227 i, \quad-0.44962-0.66707 i, \quad 0, \quad 2.1641, \quad 0.58808+1.12062 i$, $-0.78201, \quad-0.5172-1.5098 i, \quad 0.13148+0.42354 i, \quad-0.15008-0.87223 i, \quad-1.6534+0.1717 i$, $0.26139+0.99093 i,-3.8126+0.2532 i, 0.61422+0.01569 i,-0.40219+0.56716 i, 0.78585+1.09294 i$, $0.52651-1.06313 i, 3.4366+0.4772 i, 0.20918+0.81249 i, 0.19179-0.23502 i,-0.46509-0.63244 i$, $-0.07940+0.27067 i, \quad-0.96433+0.28726 i, \quad 0.02610-0.29483 i, \quad 0.12662-0.40894 i$, $0.39814+0.03508 i$

$1.4754, \quad-0.45185, \quad-0.26993-0.31443 i, \quad 0.9431-1.0986 i, \quad 0.90647, \quad-0.70202, \quad 1.1900$, $0.64403+0.44663 i, \quad-0.70679-0.19227 i, \quad-0.44962+0.66707 i, \quad 0, \quad 2.1641, \quad 0.58808-1.12062 i$, $-0.78201, \quad-0.5172+1.5098 i, \quad 0.13148-0.42354 i, \quad-0.15008+0.87223 i, \quad-1.6534-0.1717 i$, $0.26139-0.99093 i,-3.8126-0.2532 i, 0.61422-0.01569 i,-0.40219-0.56716 i, 0.78585-1.09294 i$, $0.52651+1.06313 i, 3.4366-0.4772 i, 0.20918-0.81249 i, 0.19179+0.23502 i,-0.46509+0.63244 i$, $-0.07940-0.27067 i, \quad-0.96433-0.28726 i, \quad 0.02610+0.29483 i, \quad 0.12662+0.40894 i$, $0.39814-0.03508 i$

$0.91505, \quad-0.72856, \quad 0.27183+0.31858 i, \quad-0.9300+1.0899 i, \quad 1.3057, \quad-0.48737, \quad 2.0032$, $-0.14161-0.38149 i, \quad-0.57789-0.19254 i, \quad-0.65685-0.95410 i, \quad 0, \quad 1.6661, \quad 1.4419-0.0115 i$, $-1.0157, \quad 0.58147-0.19814 i, \quad-0.13423+1.20011 i, \quad-0.70261+0.14143 i, \quad-0.07650+0.96588 i$, $3.1688+0.2287 i, \quad-0.24929+1.05474 i, \quad-0.57752+0.03039 i, \quad-0.114872-0.004834 i$, $0.95170+0.00885 i, \quad 0.27274+0.12463 i, \quad 0.66228+0.34284 i, \quad-0.091636-0.007210 i$, $0.05332+0.23213 i, \quad-0.42025-0.15528 i, \quad-0.04327+1.10732 i, \quad-2.8851+0.3857 i$, $0.92607-0.07941 i, 1.16570+0.08767 i, 0.42643+0.60833 i$ $0.91505, \quad-0.72856, \quad 0.27183-0.31858 i, \quad-0.9300-1.0899 i, \quad 1.3057, \quad-0.48737, \quad 2.0032$, $-0.14161+0.38149 i, \quad-0.57789+0.19254 i, \quad-0.65685+0.95410 i, \quad 0, \quad 1.6661, \quad 1.4419+0.0115 i$, $-1.0157, \quad 0.58147+0.19814 i, \quad-0.13423-1.20011 i, \quad-0.70261-0.14143 i, \quad-0.07650-0.96588 i$, $3.1688-0.2287 i, \quad-0.24929-1.05474 i, \quad-0.57752-0.03039 i, \quad-0.114872+0.004834 i$, $0.95170-0.00885 i, \quad 0.27274-0.12463 i, \quad 0.66228-0.34284 i, \quad-0.091636+0.007210 i$, $0.05332-0.23213 i, \quad-0.42025+0.15528 i, \quad-0.04327-1.10732 i, \quad-2.8851-0.3857 i$, $0.92607+0.07941 i, 1.16570-0.08767 i, 0.42643-0.60833 i$ 
$0.91505, \quad-0.72856, \quad-0.27183-0.31858 i, \quad 0.9300-1.0899 i, \quad 1.3057, \quad-0.48737, \quad 2.0032$, $0.14161+0.38149 i,-0.57789-0.19254 i, 0.65685+0.95410 i, 0,1.6661,-1.4419+0.0115 i,-1.0157$, $0.58147-0.19814 i, 0.13423-1.20011 i,-0.70261+0.14143 i, 0.07650-0.96588 i,-3.1688-0.2287 i$, $0.24929-1.05474 i, 0.57752-0.03039 i, 0.114872+0.004834 i, 0.95170+0.00885 i, 0.27274+0.12463 i$, $-0.66228-0.34284 i, \quad 0.091636+0.007210 i, \quad 0.05332+0.23213 i, \quad-0.42025-0.15528 i$, $0.04327-1.10732 i, 2.8851-0.3857 i, 0.92607-0.07941 i, 1.16570+0.08767 i,-0.42643-0.60833 i$,

$0.91505, \quad-0.72856, \quad-0.27183+0.31858 i, \quad 0.9300+1.0899 i, \quad 1.3057, \quad-0.48737, \quad 2.0032$, $0.14161-0.38149 i,-0.57789+0.19254 i, 0.65685-0.95410 i, 0,1.6661,-1.4419-0.0115 i,-1.0157$, $0.58147+0.19814 i, 0.13423+1.20011 i,-0.70261-0.14143 i, 0.07650+0.96588 i,-3.1688+0.2287 i$, $0.24929+1.05474 i, 0.57752+0.03039 i, 0.114872-0.004834 i, 0.95170-0.00885 i, 0.27274-0.12463 i$, $-0.66228+0.34284 i, \quad 0.091636-0.007210 i, \quad 0.05332-0.23213 i, \quad-0.42025+0.15528 i$, $0.04327+1.10732 i, 2.8851+0.3857 i, 0.92607+0.07941 i, 1.16570-0.08767 i,-0.42643+0.60833 i$,

$0.83084, \quad-0.80240, \quad 0.54517+0.28351 i, \quad-0.86630+0.45050 i, \quad 0.82640, \quad-0.77005, \quad 1.2825$, $-0.63500-0.65516 i, \quad-0.62821-0.25417 i, \quad 0.21863-0.73889 i, \quad 0, \quad 1.4650, \quad 0.07479+0.15346 i$, $-1.1552, \quad 0.19925+0.77645 i, \quad-0.39963+1.01146 i, \quad-0.55839+0.66064 i, \quad 0.56405+0.67208 i$, $0.49305+0.88504 i, \quad 0.47057+0.91614 i, \quad-0.114229-0.033999 i, \quad-0.03362+0.17079 i$, $1.07828-0.23571 i, \quad 0.43204+0.65475 i, \quad-0.15165+0.37036 i, \quad-0.00587+0.19230 i$, $0.05368+0.63486 i, \quad-0.59003+0.43800 i, \quad 0.12237+0.92941 i, \quad-0.073227+0.088523 i$, $0.72886+0.09120 i, 0.87484+0.67036 i,-0.13807+0.40776 i$

$0.83084, \quad-0.80240, \quad 0.54517-0.28351 i, \quad-0.86630-0.45050 i, \quad 0.82640, \quad-0.77005, \quad 1.2825$, $-0.63500+0.65516 i, \quad-0.62821+0.25417 i, \quad 0.21863+0.73889 i, \quad 0, \quad 1.4650, \quad 0.07479-0.15346 i$, $-1.1552, \quad 0.19925-0.77645 i, \quad-0.39963-1.01146 i, \quad-0.55839-0.66064 i, \quad 0.56405-0.67208 i$, $0.49305-0.88504 i, \quad 0.47057-0.91614 i, \quad-0.114229+0.033999 i, \quad-0.03362-0.17079 i$, $1.07828+0.23571 i, \quad 0.43204-0.65475 i, \quad-0.15165-0.37036 i, \quad-0.00587-0.19230 i$, $0.05368-0.63486 i, \quad-0.59003-0.43800 i, \quad 0.12237-0.92941 i, \quad-0.073227-0.088523 i$, $0.72886-0.09120 i, 0.87484-0.67036 i,-0.13807-0.40776 i$,

$0.83084, \quad-0.80240, \quad-0.54517-0.28351 i, \quad 0.86630-0.45050 i, \quad 0.82640, \quad-0.77005, \quad 1.2825$, $0.63500+0.65516 i, \quad-0.62821-0.25417 i, \quad-0.21863+0.73889 i, \quad 0, \quad 1.4650, \quad-0.07479-0.15346 i$, $-1.1552, \quad 0.19925+0.77645 i, \quad 0.39963-1.01146 i, \quad-0.55839+0.66064 i, \quad-0.56405-0.67208 i$, $-0.49305-0.88504 i, \quad-0.47057-0.91614 i, \quad 0.114229+0.033999 i, \quad 0.03362-0.17079 i$, $1.07828-0.23571 i, 0.43204+0.65475 i, 0.15165-0.37036 i, 0.00587-0.19230 i, 0.05368+0.63486 i$, $-0.59003+0.43800 i, \quad-0.12237-0.92941 i, \quad 0.073227-0.088523 i, \quad 0.72886+0.09120 i$, $0.87484+0.67036 i, 0.13807-0.40776 i$,

$0.83084, \quad-0.80240, \quad-0.54517+0.28351 i, \quad 0.86630+0.45050 i, \quad 0.82640, \quad-0.77005, \quad 1.2825$, $0.63500-0.65516 i, \quad-0.62821+0.25417 i, \quad-0.21863-0.73889 i, \quad 0, \quad 1.4650, \quad-0.07479+0.15346 i$, $-1.1552, \quad 0.19925-0.77645 i, \quad 0.39963+1.01146 i, \quad-0.55839-0.66064 i, \quad-0.56405+0.67208 i$, $-0.49305+0.88504 i, \quad-0.47057+0.91614 i, \quad 0.114229-0.033999 i, \quad 0.03362+0.17079 i$, $1.07828+0.23571 i, 0.43204-0.65475 i, 0.15165+0.37036 i, 0.00587+0.19230 i, 0.05368-0.63486 i$, $-0.59003-0.43800 i, \quad-0.12237+0.92941 i, \quad 0.073227+0.088523 i, \quad 0.72886-0.09120 i$, $0.87484-0.67036 i, 0.13807+0.40776 i$,

$0.39164, \quad-1.7022, \quad-0.35103-0.95729 i, \quad 0.20259-0.55248 i, \quad 0.38227, \quad-1.6647, \quad 1.1443$, $0.0237+1.6373 i, \quad 0.16720-0.16022 i, \quad 0.09765+0.99081 i, \quad 0, \quad 1.5031, \quad-0.53246-0.03874 i$, $-1.1259, \quad 0.21939-0.79376 i, \quad-0.26452-1.01231 i, \quad 0.80162-0.64935 i, \quad-0.43261-1.28844 i$, $-0.13172-0.75084 i, \quad-0.13792-0.80345 i, \quad-0.2954-3.4158 i, \quad 0.75140-0.93916 i$, $-0.15858-0.75731 i, \quad 0.61414+0.02289 i, \quad 0.17208-1.00307 i, \quad 0.5605-2.5543 i, 0.77415+0.28436 i$, $-1.24006-0.20187 i, \quad-0.0941-2.1116 i, \quad 0.41179-0.61812 i, \quad-0.61289-0.14385 i$, $0.00420+0.62552 i, 0.45087-1.21007 i$, 
$0.39164, \quad-1.7022, \quad-0.35103+0.95729 i, \quad 0.20259+0.55248 i, \quad 0.38227, \quad-1.6647, \quad 1.1443$, $0.0237-1.6373 i, \quad 0.16720+0.16022 i, \quad 0.09765-0.99081 i, \quad 0, \quad 1.5031, \quad-0.53246+0.03874 i$, $-1.1259, \quad 0.21939+0.79376 i, \quad-0.26452+1.01231 i, \quad 0.80162+0.64935 i, \quad-0.43261+1.28844 i$, $-0.13172+0.75084 i, \quad-0.13792+0.80345 i, \quad-0.2954+3.4158 i, \quad 0.75140+0.93916 i$, $-0.15858+0.75731 i, \quad 0.61414-0.02289 i, \quad 0.17208+1.00307 i, \quad 0.5605+2.5543 i, \quad 0.77415-0.28436 i$, $-1.24006+0.20187 i, \quad-0.0941+2.1116 i, \quad 0.41179+0.61812 i, \quad-0.61289+0.14385 i$, $0.00420-0.62552 i, 0.45087+1.21007 i$,

$0.39164, \quad-1.7022, \quad 0.35103+0.95729 i, \quad-0.20259+0.55248 i, \quad 0.38227, \quad-1.6647, \quad 1.1443$, $-0.0237-1.6373 i, 0.16720-0.16022 i,-0.09765-0.99081 i, 0,1.5031,0.53246+0.03874 i,-1.1259$, $0.21939-0.79376 i, 0.26452+1.01231 i, 0.80162-0.64935 i, 0.43261+1.28844 i, 0.13172+0.75084 i$, $0.13792+0.80345 i, 0.2954+3.4158 i,-0.75140+0.93916 i,-0.15858-0.75731 i, 0.61414+0.02289 i$, $-0.17208+1.00307 i,-0.5605+2.5543 i, 0.77415+0.28436 i,-1.24006-0.20187 i, 0.0941+2.1116 i$, $-0.41179+0.61812 i,-0.61289-0.14385 i, 0.00420+0.62552 i,-0.45087+1.21007 i$,

$0.39164, \quad-1.7022, \quad 0.35103-0.95729 i, \quad-0.20259-0.55248 i, \quad 0.38227, \quad-1.6647, \quad 1.1443$, $-0.0237+1.6373 i, 0.16720+0.16022 i,-0.09765+0.99081 i, 0,1.5031,0.53246-0.03874 i,-1.1259$, $0.21939+0.79376 i, 0.26452-1.01231 i, 0.80162+0.64935 i, 0.43261-1.28844 i, 0.13172-0.75084 i$, $0.13792-0.80345 i, 0.2954-3.4158 i,-0.75140-0.93916 i,-0.15858+0.75731 i, 0.61414-0.02289 i$, $-0.17208-1.00307 i,-0.5605-2.5543 i, 0.77415-0.28436 i,-1.24006+0.20187 i, 0.0941-2.1116 i$, $-0.41179-0.61812 i,-0.61289+0.14385 i, 0.00420-0.62552 i,-0.45087-1.21007 i$,

$0.81486, \quad-0.81813, \quad 0.79755-0.60073 i, \quad-0.47999-0.36154 i, \quad 0.78430, \quad-0.81138, \quad 0.41009$, $-0.44206-1.09721 i, \quad-0.34785+0.02123 i, \quad 0.41308-1.00557 i, \quad 0, \quad 1.4888, \quad-0.22666+0.09476 i$, $-1.1367, \quad-0.69448+0.34912 i, \quad-0.45294+1.25709 i, \quad 0.36740+0.11056 i, \quad 0.27722+0.88306 i$, $-0.2915+2.1764 i, \quad-0.3057+2.3029 i, \quad-1.7000-0.4665 i, \quad 1.3091+0.9847 i, \quad 0.13999+0.46494 i$, $0.92298+0.19622 i, \quad 0.90835+1.06028 i, \quad 0.4322+1.8622 i, \quad 0.27019-0.27159 i,-0.59122+0.18392 i$, $0.00432+1.26521 i, 0.96442+0.86007 i,-0.64767-0.00816 i,-0.11705-0.57289 i, 1.1066+1.0690 i$,

$0.81486, \quad-0.81813, \quad 0.79755+0.60073 i, \quad-0.47999+0.36154 i, \quad 0.78430, \quad-0.81138, \quad 0.41009$, $-0.44206+1.09721 i, \quad-0.34785-0.02123 i, \quad 0.41308+1.00557 i, \quad 0, \quad 1.4888, \quad-0.22666-0.09476 i$, $-1.1367, \quad-0.69448-0.34912 i, \quad-0.45294-1.25709 i, \quad 0.36740-0.11056 i, \quad 0.27722-0.88306 i$, $-0.2915-2.1764 i, \quad-0.3057-2.3029 i, \quad-1.7000+0.4665 i, \quad 1.3091-0.9847 i, \quad 0.13999-0.46494 i$, $0.92298-0.19622 i, 0.90835-1.06028 i, 0.4322-1.8622 i, 0.27019+0.27159 i,-0.59122-0.18392 i$, $0.00432-1.26521 i, 0.96442-0.86007 i,-0.64767+0.00816 i,-0.11705+0.57289 i, 1.1066-1.0690 i$,

(B.56)

$0.81486, \quad-0.81813, \quad-0.79755+0.60073 i, \quad 0.47999+0.36154 i, \quad 0.78430, \quad-0.81138, \quad 0.41009$, $0.44206+1.09721 i,-0.34785+0.02123 i,-0.41308+1.00557 i, 0,1.4888,0.22666-0.09476 i,-1.1367$, $-0.69448+0.34912 i, 0.45294-1.25709 i, 0.36740+0.11056 i,-0.27722-0.88306 i, 0.2915-2.1764 i$, $0.3057-2.3029 i, \quad 1.7000+0.4665 i, \quad-1.3091-0.9847 i, \quad 0.13999+0.46494 i, \quad 0.92298+0.19622 i$, $-0.90835-1.06028 i, \quad-0.4322-1.8622 i, \quad 0.27019-0.27159 i, \quad-0.59122+0.18392 i$, $-0.00432-1.26521 i, \quad-0.96442-0.86007 i, \quad-0.64767-0.00816 i, \quad-0.11705-0.57289 i$,

$-1.1066-1.0690 i$

$0.81486, \quad-0.81813, \quad-0.79755-0.60073 i, \quad 0.47999-0.36154 i, \quad 0.78430, \quad-0.81138, \quad 0.41009$, $0.44206-1.09721 i,-0.34785-0.02123 i,-0.41308-1.00557 i, 0,1.4888,0.22666+0.09476 i,-1.1367$, $-0.69448-0.34912 i, 0.45294+1.25709 i, 0.36740-0.11056 i,-0.27722+0.88306 i, 0.2915+2.1764 i$, $0.3057+2.3029 i, \quad 1.7000-0.4665 i, \quad-1.3091+0.9847 i, \quad 0.13999-0.46494 i, \quad 0.92298-0.19622 i$, $-0.90835+1.06028 i, \quad-0.4322+1.8622 i, \quad 0.27019+0.27159 i, \quad-0.59122-0.18392 i$, $-0.00432+1.26521 i, \quad-0.96442+0.86007 i, \quad-0.64767+0.00816 i, \quad-0.11705+0.57289 i$, $-1.1066+1.0690 i$. 
Open Access. This article is distributed under the terms of the Creative Commons Attribution License (CC-BY 4.0), which permits any use, distribution and reproduction in any medium, provided the original author(s) and source are credited.

\section{References}

[1] A. Sen, Arithmetic of quantum entropy function, JHEP 08 (2009) 068 [arXiv:0903.1477] [INSPIRE].

[2] A. Dabholkar, J. Gomes, S. Murthy and A. Sen, Supersymmetric index from black hole entropy, JHEP 04 (2011) 034 [arXiv:1009.3226] [INSPIRE].

[3] A. Sen, How do black holes predict the sign of the Fourier coefficients of Siegel modular forms?, Gen. Rel. Grav. 43 (2011) 2171 [arXiv: 1008.4209] [INSPIRE].

[4] K. Bringmann and S. Murthy, On the positivity of black hole degeneracies in string theory, Commun. Num. Theor Phys. 07 (2013) 15 [arXiv:1208.3476] [INSPIRE].

[5] F. Denef and G.W. Moore, Split states, entropy enigmas, holes and halos, JHEP 11 (2011) 129 [hep-th/0702146] [INSPIRE].

[6] J. Manschot, B. Pioline and A. Sen, A fixed point formula for the index of multi-centered $N=2$ black holes, JHEP 05 (2011) 057 [arXiv:1103.1887] [INSPIRE].

[7] I. Bena, M. Berkooz, J. de Boer, S. El-Showk and D. Van den Bleeken, Scaling BPS solutions and pure-Higgs states, JHEP 11 (2012) 171 [arXiv:1205.5023] [INSPIRE].

[8] S.-J. Lee, Z.-L. Wang and P. Yi, Quiver invariants from intrinsic Higgs states, JHEP 07 (2012) 169 [arXiv:1205.6511] [INSPIRE].

[9] S.-J. Lee, Z.-L. Wang and P. Yi, BPS states, refined indices and quiver invariants, JHEP 10 (2012) 094 [arXiv:1207.0821] [INSPIRE].

[10] J. Manschot, B. Pioline and A. Sen, From black holes to quivers, JHEP 11 (2012) 023 [arXiv:1207.2230] [INSPIRE].

[11] J. Manschot, B. Pioline and A. Sen, On the Coulomb and Higgs branch formulae for multi-centered black holes and quiver invariants, JHEP 05 (2013) 166 [arXiv:1302.5498] [INSPIRE].

[12] A. Sen, Walls of marginal stability and dyon spectrum in $N=4$ supersymmetric string theories, JHEP 05 (2007) 039 [hep-th/0702141] [INSPIRE].

[13] A. Dabholkar, D. Gaiotto and S. Nampuri, Comments on the spectrum of CHL dyons, JHEP 01 (2008) 023 [hep-th/0702150] [INSPIRE].

[14] A. Sen, Two centered black holes and $N=4$ dyon spectrum, JHEP 09 (2007) 045 [arXiv:0705.3874] [INSPIRE].

[15] M.C.N. Cheng and E. Verlinde, Dying dyons don't count, JHEP 09 (2007) 070 [arXiv:0706.2363] [INSPIRE].

[16] A. Sen, $N=8$ dyon partition function and walls of marginal stability, JHEP 07 (2008) 118 [arXiv: 0803.1014] [INSPIRE].

[17] A. Chowdhury, R.S. Garavuso, S. Mondal and A. Sen, BPS state counting in $N=8$ supersymmetric string theory for pure D-brane configurations, JHEP 10 (2014) 186 [arXiv:1405.0412] [INSPIRE]. 
[18] E. Witten, Constraints on supersymmetry breaking, Nucl. Phys. B 202 (1982) 253 [InSPIRE].

[19] S. Sethi and M. Stern, Invariance theorems for supersymmetric Yang-Mills theories, Adv. Theor. Math. Phys. 4 (2000) 487 [hep-th/0001189] [InSPIRE].

[20] J.M. Maldacena, G.W. Moore and A. Strominger, Counting BPS black holes in toroidal type II string theory, hep-th/9903163 [INSPIRE].

[21] D. Shih, A. Strominger and X. Yin, Counting dyons in $N=8$ string theory, JHEP 06 (2006) 037 [hep-th/0506151] [INSPIRE].

[22] F. Denef, Quantum quivers and Hall/hole halos, JHEP 10 (2002) 023 [hep-th/0206072] [INSPIRE].

[23] C. Bachas and E. Kiritsis, $F^{4}$ terms in $N=4$ string vacua, Nucl. Phys. Proc. Suppl. 55B (1997) 194 [hep-th/9611205] [InSPIRE].

[24] A. Gregori, E. Kiritsis, C. Kounnas, N.A. Obers, P.M. Petropoulos and B. Pioline, $R^{2}$ corrections and nonperturbative dualities of $N=4$ string ground states, Nucl. Phys. B 510 (1998) 423 [hep-th/9708062] [INSPIRE].

[25] S. Banerjee, R.K. Gupta and A. Sen, Logarithmic corrections to extremal black hole entropy from quantum entropy function, JHEP 03 (2011) 147 [arXiv: 1005.3044] [INSPIRE].

[26] S. Banerjee, R.K. Gupta, I. Mandal and A. Sen, Logarithmic corrections to $N=4$ and $N=8$ black hole entropy: a one loop test of quantum gravity, JHEP 11 (2011) 143 [arXiv: 1106.0080] [INSPIRE].

[27] A. Dabholkar, J. Gomes and S. Murthy, Quantum black holes, localization and the topological string, JHEP 06 (2011) 019 [arXiv: 1012.0265] [INSPIRE].

[28] A. Dabholkar, J. Gomes and S. Murthy, Localization \& exact holography, JHEP 04 (2013) 062 [arXiv:1111.1161] [INSPIRE].

[29] A. Dabholkar, J. Gomes and S. Murthy, Nonperturbative black hole entropy and Kloosterman sums, JHEP 03 (2015) 074 [arXiv: 1404.0033] [INSPIRE].

[30] W. Decker, G.-M. Greuel, G. Pfister and H. Schönemann, Singular 4-0-2 - a computer algebra system for polynomial computations, http://www.singular.uni-kl.de, (2015).

[31] D.R. Grayson and M.E. Stillman, Macaulay2, a software system for research in algebraic geometry, http://www.math.uiuc.edu/Macaulay2/, (2015).

[32] Wolfram Research Inc., Mathematica, version 10.3, Champaign IL U.S.A. (2015).

[33] S.D. Mathur, The fuzzball proposal for black holes: an elementary review, Fortsch. Phys. 53 (2005) 793 [hep-th/0502050] [INSPIRE].

[34] S.D. Mathur, Fuzzballs and the information paradox: a summary and conjectures, arXiv:0810.4525 [INSPIRE].

[35] I. Bena and N.P. Warner, Black holes, black rings and their microstates, Lect. Notes Phys. 755 (2008) 1 [hep-th/0701216] [INSPIRE].

[36] K. Skenderis and M. Taylor, The fuzzball proposal for black holes, Phys. Rept. 467 (2008) 117 [arXiv:0804.0552] [INSPIRE].

[37] V. Balasubramanian, J. de Boer, S. El-Showk and I. Messamah, Black holes as effective geometries, Class. Quant. Grav. 25 (2008) 214004 [arXiv:0811.0263] [INSPIRE]. 
[38] O. Lunin, Bubbling geometries for $A d S_{2} \times S^{2}$, JHEP 10 (2015) 167 [arXiv:1507.06670] [INSPIRE].

[39] A. Sen, State operator correspondence and entanglement in $A d S_{2} / C F T_{1}$, Entropy 13 (2011) 1305 [arXiv: 1101.4254] [INSPIRE].

[40] D. Friedan, E.J. Martinec and S.H. Shenker, Conformal invariance, supersymmetry and string theory, Nucl. Phys. B 271 (1986) 93 [INSPIRE]. 